\title{
Manga Dante. Comunicazione interculturale e tradizione figurativa in Dante Shinkyoku di Gō Nagai
}

\author{
Mario Tirino \\ Università di Salerno \\ mario.tirino@gmail.com
}

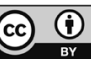

\section{Riassunto}

Gō Nagai è universalmente riconosciuto come uno dei più importanti mangaka della storia del fumetto nipponico. Autore di storie legate a personaggi cruciali tanto per l'immaginario orientale quanto per quello occidentale, quali Mazinga Z e Getter Robot, Gō Nagai (vero nome Kiyoshi Nagai), per buona parte della propria carriera, intrattiene un dialogo con la cultura italiana e, in particolare, con la Divina Commedia di Dante. Da un lato, Nagai si ispira al poema dantesco, adattandone atmosfere, umori e singoli personaggi in saghe come Mao Dante o Devilman (il cui protagonista è dichiaratamente ispirato al Lucifero dantesco). Dall'altro, Nagai accoglie la sfida di trasporre interamente la Commedia in un'opera a fumetti di poderose dimensioni, Dante Shinkyoku (3 voll., 1993-1994). Lo sforzo creativo del manga$k a$ svela due importanti qualità del medium fumettistico: la capacità di fungere da strumento di comunicazione e traduzione interculturale, in grado di avvicinare e rendere percepibili culture apparentemente distanti, e la abilità nel rimediare, negli spazi altamente immersivi della lettura di nuvole e vignette, altre tradizioni figurative - nello specifico l'eredità dell'illustrazione di Gustave Doré, vero filtro della poetica dantesca nei medium audiovisivi.

Parole chiave: Dante, Gō Nagai, Gustave Doré, comunicazione interculturale, rimediazione

\begin{abstract}
Gō Nagai is universally recognized as one of the most important mangaka in the history of Japanese comics. Author of stories related to crucial characters for both the Eastern and Western imaginary, such as Mazinga Z, Gō Nagai (real name Kiyoshi Nagai), for a good part of his career, entertains a dialogue with Italian culture and, in particular, with the Divine Comedy by Dante. On the one hand, Nagai is inspired by the Dante's poem, adapting atmospheres, moods and individual characters in sagas like Mao Dante and Devilman (whose protagonist is openly inspired by the Dante's Lucifer). On the other hand, Nagai accepts the challenge of completely transposing the play into a comic book of mighty dimensions, Dante Shinkyoku (3 voll., I993-1994). The creative effort of the Japanese mangaka reveals two important qualities of the comic book medium: its ability to act as an instrument of communication and intercultural translation, able to bring together and make perceivable cultures seemingly distant, and to reconfigure, in the highly immersive spaces of reading clouds and cartoons, other figurative traditions - specifically the legacy of illustration by Gustave Doré, a true "filter" of Dante's poetry in audiovisual mediums.
\end{abstract}

Keywords: Dante, Gō Nagai, Gustave Doré, intercultural comunication, remediation 


\section{Premessa i. La Divina Commedia e l'iconopoiesi}

T a Divina Commedia di Dante ha goduto negli ultimi venti anni di una notevole mole di traduzioni, omaggi, citazioni nella cultura popolare contemporanea, passando attraverso diversi tipi di media (film, serie televisive, romanzi, videogame); tra questi il fumetto occupa un posto privilegiato. La quantità di prodotti culturali, di natura prevalentemente iconica, rende necessaria una premessa circa le potenzialità di generare immagini che il poema dantesco ha manifestato nel corso dei secoli. Nell'imponente corpus letterario della Commedia alberga una sovrabbondante mole di dati, che restituiscono un "quadro assai preciso delle forme (umane, geografiche, paesaggistiche, vegetali ed animali)", con un corredo "di dettagli sulla flora e sulla fauna, sulle costellazioni, nonché sulle condizioni di luce (il sorgere o il tramontare del sole), sugli agenti atmosferici (piogge, nebbie) e, in generale, sulle condizioni stesse di visibilità" (Amendola, Tirino 2016: I2). Tre sono i principali elementi di questa potenza iconopoietica della Commedia: il paesaggio, le luci e i colori, la similitudine.

Le strutture morfologiche del paesaggio svolgono un ruolo fondamentale, oltre che come quinta o sfondo (Bandirali 1999: 13), anche come spazio fisico-simbolico in grado di condensare i sentimenti di chi lo attraversa o lo abita. In questo senso, le caratteristiche dei luoghi attraversati tra Inferno, Purgatorio e Paradiso, sono indicative delle forze emotive di Dante e delle anime che vi transitano.

Per quanto attiene ai colori e alle luci, basti ricordare la minuziosa attenzione riservata alle proprietà luminose degli ambienti, ulteriormente testimoniata dall'accuratezza delle descrizioni cromatiche. Le cromìe caricano affettivamente le scene, costruendo ambienti simbolico-allegorici: ogni cantica è caratterizzata dalla prevalenza di alcune tonalità e sfumature (De Angelis 1967); così, nel Purgatorio domina un lucore tenue e sfumato, mentre il Paradiso è puntellato da bagliori splendenti e riflessi accecanti. Tuttavia è nell'inferno che i colori dispiegano compiutamente la loro qualità metaforica: "possiamo individuare tre zone nel baratro infernale che si distinguono proprio per i suoi diversi effetti cromatici di base, in cui dominano rispettivamente il nero, il rosso e l'azzurro. Le zone cromatiche concordano con i tre tipi di peccatori: gli incontinenti, i violenti e i fraudolenti” (Hofer 20I0: 45).

Il terzo elemento che compone "la strategia iconopoietica dantesca è la similitudine, che conferisce a concetti e ideali una sostanza visiva (...). Dante popola le sue pagine di figure veraci, dalla intensa carica sensoriale, impiegate per guidare il lettore in una significazione che, da verbale, si fa eminentemente verbo-visiva, insieme di visione e visionarietà" (Amendola, Tirino 2016: 13). È con questo ricco materiale che si confrontano tutti gli autori interessati a tradurre in immagini le terzine dantesche. 


\section{Premessa 2. Dante in Giappone}

Il presente saggio vuole indagare le forme e i processi socioculturali attraverso i quali la Commedia dantesca viene rielaborata nell'opera del mangaka Gō Nagai, tra i più importanti autori di fumetti e anime nipponici. Tuttavia, prima di entrare nel cuore dell'analisi, occorre ricostruire, seppure molto sinteticamente, come l'opera di Dante arriva in terra giapponese. Com'è noto, a causa dell'isolazionismo dello shogunato Tokugawa (I600-I866), le grandi opere del patrimonio letterario occidentale arrivano a singhiozzo e con molto ritardo. Grazie ad alcuni studi (Kure 1965, Nogami 1965, Fujisawa 1965, Fujitani 2000, Iwakura 200I, Capponcelli 20I4, Doi 2016), è oggi possibile ricostruire, con maggiore accuratezza, i passaggi che hanno contribuito alla diffusione e alla fortuna della Commedia in Giappone. Con l'avvento dell'era Meiji (I868 - I9I2) e la relativa, moderata apertura alle culture occidentali, tra i primi ad avvicinarsi a Dante è lo psichiatra Uchimura Kanzo, protagonista fin dal 1898 di affollate conferenze, il cui principale intento è analizzare la Commedia nel quadro di un inedito interesse per il Cristianesimo quale forma di resistenza alle derive della modernizzazione (Capponcelli 20I4). Le conferenze di Uchimura ispirano gli studi dello scrittore e critico Makucho Masamune, interessato soprattutto alla centralità dell'Io (che lo porta a un collegamento con l'opera del drammaturgo svedese Johan August Strindberg) e a una visione romantica della spiritualità medievale (Capponcelli 20I4: 85). Negli stessi anni il poeta e traduttore Ueda Bin dà alle stampe la prima monografia su Dante in Giappone (Il vate Dante, I90I). Tuttavia va ricordato che questi intellettuali leggevano la Commedia in traduzioni giapponesi dall'inglese o dal tedesco. Solo tra il I9I4 e il I9I6 arriva la prima traduzione direttamente dal testo originale, opera dell'italianista Yamakawa Heisaburo. Negli anni Venti e Trenta l'opera di Dante è sia oggetto di intensi studi - di carattere critico-letterario (le riflessioni di Kiyoteru Hanada sul conflitto tra politica e letteratura che domina la vita e l'opera dell'Alighieri) e filosofico (l'analisi comparata di Dante e Nietzsche di Jiro Abe) -, sia fonte di ispirazione per vari letterati, come la poetessa Akiko Yosano, autrice di un componimento ispirato all'Inferno (I92I) e lo scrittore Ryūnosuke Akutagawa, il cui racconto Haguruma (La ruota dentata, 1927) è evidentemente influenzato da una lettura dell'Inferno dantesco come specchio del male terreno (un'interpretazione che coniuga Dante e Strindberg, come in Masamune). Fondamentale per la circolazione della Commedia è l'opera di Ogai Mori, al quale si deve il termine giapponese, di nuovo conio, Shinkyoku, con cui ancora oggi è conosciuto il poema sull'isola: traducendo in giapponese il romanzo L'improvvisatore (I835) di Hans Christian Andersen, il cui protagonista rinviene a un certo punto una copia del poema, Mori per tradurne il titolo combina i due ideogrammi 
"Dio" (shin) e "canto di gusto" (kyoku) (Doi 20I6). Il grande impulso alla circolazione della Commedia tra le élite di intellettuali e scrittori nipponici è fornito, però, dal fiorire di nuove traduzioni: dopo quella di Heisaburo, vanno citate quelle di Masaki Nakayama (1917), Sofu Taketomo (1923) e Choko Ikuta (1929). La fortuna di queste traduzioni è tale che Kure (1965: I7), a metà degli anni Sessanta, scrive, forse troppo entusiasticamente, che "9 scrittori contemporanei su IO, uomini di alta cultura, hanno letto, in Giappone, se non per intero, almeno in parte la grandiosa opera di Dante Alighieri". Alle traduzioni dell'anteguerra, se ne aggiungono altre, ugualmente rilevanti per la conoscenza della Commedia in Giappone, nel dopoguerra: ricordiamo quelle di Soichi Nogami (1964), cui va il grande merito di aver reso concetti cristiani in una cultura scintoista come quella nipponica, di Sukehiro Hirakawa (1966), Itsuo Miura (1970-72), Bunsho Jugaku (1974-76) e Motoaki Hara (2015). La testimonianza del fatto che la Commedia ha esercitato un fascino duraturo sugli autori giapponesi arriva anche dal romanzo Natsukashii toshi e no tegami (Gli anni della nostalgia, 1987) di Kenzaburo Oe, in cui abbondano le citazioni dell'opera attraverso il personaggio di Gii (Doi 20I6).

\section{Gō Nagai. Avvisaglie dantesche: Mao Dante (I97I) e DeVilman (I972)}

Gō Nagai può essere considerato come uno degli autori che più a lungo e più a fondo hanno inciso nell'immaginario popolare, occidentale e orientale. Le sue opere possono essere considerate come esempi paradigmatici della natura ormai globalizzata della cultura otaku (Ito, Okabe, Tsuji 20I2):

La produzione fumettistica nipponica rappresenta, ad oggi, un fenomeno di portata globale, grazie al quale elementi del J-pop, il volto più mediatico della pop-culture giapponese - una costruzione solo parzialmente inconscia, saldamente radicata nel patrimonio culturale condiviso, fondata su una commistione di schegge di diverse tradizioni culturali, la cui provenienza è sostanzialmente transnazionale - rimbalzano costantemente tra Oriente ed Occidente, in un gioco di specchi che rende quanto mai arduo seguire il flusso delle differenti tradizioni che hanno concorso alla creazione di una delle culture giovanili maggiormente diffuse e rappresentate al mondo (Scilabra 2015: 92-93).

La J-Pop, nutrita da manga, anime, giochi e videogames, si fonda su un aspetto centrale che occorre adeguatamente evidenziare ai fini di un'analisi appropriata del rapporto di Gō Nagai con la tradizione culturale occidentale: ci riferiamo al sincretismo con cui gli autori giapponesi tendono ad inglobare elementi culturali estranei, riformulati e assorbiti nell'immaginario collettivo attraverso linguaggi, forme e modalità più accessibili al pubblico nazionale (Pellitteri 2008, Bouissou 20IO, 20II). Se ciò è vero per l'uso che degli elementi 
classici Gō Nagai fa nella trilogia mecha, costituita da Mazinger $Z$ (1972), Great Mazinger (1974) e Ufo Robot Grendizer (1975) (Gilson 1998, Clements, McCarthy 2006), in cui "l'alterità - ed un'alterità decisamente ostile per di più - è rappresentata dall'eredità del classico, nella quale è difficile non vedere una metafora per il mondo occidentale" (Scilabra 2015: IO2), lo è tanto più per il ruolo svolto dalla Commedia dantesca, nella versione illustrata da Doré, per i due lavori di Gō Nagai, Mao Dante (197I) e Devilman (1972). Come conferma lui stesso in tre diverse occasioni, quasi con le medesime parole, per l'autore, intervistato da Prisco (2007), Danesi (2016) e dalla casa editrice Edizioni BD per gli extra di Devilman Omnibus (2017), il fascino della Commedia si manifesta in due diverse modalità. Da un lato, c'è l'incontro con il disegno di Doré, che rappresenta per Gō Nagai una vera epifania: questa lettura giovanile instilla in lui il desiderio di misurarsi con l'iconografia dell'illustratore francese - obiettivo raggiunto solo con la vera e propria trasposizione della Commedia nella trilogia Dante Shinkyoku (1994). Dall'altro lato, c'è il ricco materiale simbolico e allegorico dell'opera dantesca, che, nelle forme sincretiche della cultura fumettistica nipponica, viene trasfuso in Mao Dante, Shin Mao Dante (l'arco narrativo con cui, tra il 2002 e il 2003 , si concludono le avventure di Ryo Utsugi, e che è pertanto successivo a Dante Shinkyoku) e Devilman.

I livelli in cui la ricchezza simbolico-allegorica della Commedia dantesca si riflette nella produzione gonagaiana, che precede e segue la trasposizione vera e propria, sono sei.

Un primo livello riguarda il carattere dei personaggi, tutti dotati di un'inesauribile forza di volontà, di un senso di abnegazione che, in ragione della missione loro affidata, li conduce ad affrontare e superare ogni ostacolo. Come Dante, che deve combattere contro le sue paure prima di superare la "selva oscura" (Inf. I), così il protagonista di Mao Dante è un giovane studente, Ryo Utsugi, il quale deve affrontare una sfida enorme: gli è chiesto, infatti, di fondersi con il corpo di un'orrenda bestia demoniaca, il Re Demone Dante, che sembra, inizialmente, essere a capo di forze maligne e assassine. Analogamente, in Devilman, il timidissimo, terrorizzato e impacciato Akira Fudo deve compiere una scelta che gli cambierà per sempre la vita: il suo più caro amico Ryo gli chiede di diventare un demone per combattere, dall'interno, gli altri demoni e salvare l'umanità. Nella visione gonagaiana, il sacrificio di Ryo, quello di Akira e quello di Dante possono essere assimilati, in quanto a tutti e tre è chiesto di votarsi ad una causa senza esitazioni, nel nome del boushido, la via del guerriero, che ricorre con frequenza nei manga nipponici (Castello, Scilabra 20I5). In Mao Dante, il momento decisivo della scelta si può ravvisare nella pagina intera in cui Ryo, nel mezzo di una tormenta di neve, deve attraversare una sorta di tunnel, per obbedire al richiamo del demone che alberga sotto le pareti rocciose dell'Himalaya (Fig. Ia). In Devilman, invece, Gō Nagai 
realizza un potente primissimo piano di Akira (Fig. Ib), che, in lacrime, realizza che la sua esistenza terrena cambierà irreparabilmente dopo la scelta di trasformarsi.

Come scrive Raffaelli (1993: 37), si tratta di un topos del fumetto giapponese, in cui "i personaggi sono invasi da una forza cieca, incontrollabile, che viene dall'esterno e che non possono padroneggiare". Un secondo livello può essere rinvenuto, dunque, nello statuto interdimensionale, liminale che accomuna il Dante della Commedia e i personaggi di Ryo Utsugi e Akira Fudo: essi, infatti, sono in preda allo smarrimento provato nel passaggio da una dimensione all'altra (dal mondo terreno a quello ultraterreno, nel caso di Dante nella Divina Commedia, dall'essere umano al demone nel caso di Ryo Utsugi in Mao Dante e di Akira Fudo in Devilman). Questa sensazione, causata dall'invasione inarrestabile di forze esterne sul proprio corpo, è uno stato temporaneo, superato, appunto, attraverso la forza di volontà. In particolare, Ryo e Akira devono imparare a padroneggiare i superpoteri acquisiti con le trasformazioni dei loro corpi, da cui derivano nuove possibilità e nuovi rischi. Anche se nel ristretto spazio di questo saggio non è possibile fare un'analisi approfondita, tuttavia è abbastanza evidente che siamo in presenza di un mitologema universale - la capacità dell'eroe e del supereroe di misurarsi con l'accrescimento e la metamorfosi del proprio corpo (Frezza 1995) - che andrebbe esaminato nelle sue configurazioni puntuali rispetto ai diversi contesti socioculturali. Ci limiteremo qui a notare come proprio questo mitologema costituisca un trait d'union tra i due fumetti horror Mao Dante e Devilman e la trilogia mecha, in quanto lo stesso concetto di "super robot" può essere riconsiderato come corpo tecnologico guidato da un essere umano; con il corollario di traumi, responsabilità e potenzialità che ne derivano.

Un terzo livello riguarda l'ibridazione e talvolta l'indistinguibilità tra bene e male, in cui sono immersi tutti i personaggi gonagaiani. Come scrive Bombara (2018: I26),

Nei personaggi di Mao Dante (I97I), un umano che scopre di essere la reincarnazione di un demone potentissimo - e solo apparentemente malvagio - chiamato Dante, e di Devilman (1972), giovane che assume forme demoniche per sconfiggere dall'interno il Male e salvare la Terra, vediamo operante la commistione fra Bene e Male che informa di sé anche la successiva versione gonaghiana della Commedia, e ne costituisce la componente più innovativa; d'altra parte, l'interazione positivo/negativo è anche un elemento strutturante del genere manga.

Questa commistione tra bene e male assume talora la forma del dissidio interno, com'è evidente nelle numerose vignette in cui Ryo non riesce ad accettare le forme orribili del suo nuovo corpo di demone infernale (Fig. 2). 
Un quarto livello ha a che vedere con il recupero di personaggi della mitologia classica, presenti o meno nella Divina Commedia, all'interno di Mao Dante, Shin Mao Dante e Devilman. Questo processo socioculturale attesta l'enorme capacità della tradizione filosofica e artistica della tradizione grecoromana di stimolare una costante riflessione sul concetto di classico (Settis 2004). Occorre ribadire che, in Mao Dante e Shin Mao Dante, le figure mitologiche classiche, così come quelle attinte dall'angelologia e demonologia cristiane, sono trattate con estrema libertà creativa, nella dimensione sincretica di cui si è detto prima (Scilabra 20I5). È il caso per esempio di Medusa (Fig. 3a) che, in Mao Dante, è un demone, chiaramente ispirato ad una delle Gorgoni. $\mathrm{E}$ interessante notare che, in Shin Mao Dante, possiamo osservare una versione di Medusa tecnologicamente evoluta e attrezzata (Fig. 3b). Nella stessa serie, le entità divine "aliene", essendo pura energia, hanno la necessità di "occupare" corpi viventi, umani e non; ed è appunto, dalla loro fusione con corpi animali che nascono figure come i centauri, Tritone, guerrieri alati e così via, chiaramente attinti dalla mitologia classica (Fig. 3c). In Devilman Gō Nagai, in collaborazione con Hiroshi Koenjij, realizza un'intera storia (dal titolo $L a$ Nike di Samotracia. La bella Dea della Guerra), in cui Akira/Devilman e Ryo viaggiano nel tempo, per smascherare un demone che si spaccia per la Dea Nike e dota di armi moderne l'esercito ellenico. Come spiegano Ponticiello e Scrivo (2007), i mangaka, come gli altri artisti giapponesi, non si pongono criticamente rispetto ai materiali della cultura occidentale che assorbono; in tal modo, facendo prevalere la componente "affettiva" del rapporto con le opere, possono mixare liberamente fonti alte e fonti basse. È esattamente quanto avviene in Mao Dante, dove le suggestioni del poema convivono con espliciti riferimenti visivi ai kaiju eiga (i film con enormi mostri, come Godzilla, che si scontrano nel cuore delle metropoli) (Fig. 4), alla fantascienza americana (Dio come invasore alieno: Fig. 5) e persino all'iconografia dei supereroi americani (Ryo/Dante utilizza un'auto che fa il verso alla Batmobile: Fig. 6). Nella stessa chiave vanno lette le spericolate revisioni della tradizione biblica: i demoni (che conservano i nomi della tradizione cristiana: Lucifero, Satana e così via), in Mao Dante e Shin Mao Dante, sono gli abitanti originari della Terra, spodestati dall'ira di un Dio alieno; la pacifica città di Sodoma è distrutta dalla feroce divinità venuta dallo spazio; i giganteschi draghi e cavalli scatenati da "Dio" sono in qualche modo connessi alle figure dell'Apocalisse di San Giovanni. Altrettanta libertà si riscontra nel riutilizzo di icone del Cristianesimo: è quanto accade, ad esempio, nella stupenda storia Giovanna D'Arco nel regno demoniaco, una delle avventure che, nel corso degli anni, ha arricchito l'eterogeneo e amplissimo corpus narrativo di Devilman. Vi si narra del tentativo, ordito dai demoni, di giustiziare la giovane guerriera cristiana, per fare in modo che la Guerra dei Cent'anni tra Francia ed Inghilterra non si esaurisca. 
Solo l'intervento di Devilman scongiurerà questa eventualità. D'altronde, è lo stesso autore a ricordare, in un'intervista (Danesi 20I6), che ha desunto la maggior parte delle informazioni sulla religione cattolica dalla visione del film La Bibbia (The Bible: in the beginning..., I966) di John Huston: "stavo lavorando alle mie prime opere e avere avuto accesso all'intera mitologia del Cattolicesimo con un'opera così visionaria è stata una benedizione. Dovete pensare che tanti anni fa il Cattolicesimo non era poi così noto in Giappone, per noi era qualcosa di assolutamente esotico". Ciò conferma che l'interesse di Gō Nagai è riservato in maniera esclusiva alla ricca drammaturgia del materiale biblico, e non alle complesse implicazioni morali, spirituali, teologiche e filosofiche del testo. La storia Giovanna D'Arco nel regno demoniaco si inserisce in una serie di viaggi spaziotemporali di Devilman e Ryo, che ricomprende, oltre al già citato La Nike di Samotracia, Una tarda primavera a Vienna (che racconta l'antefatto della trasformazione di un mediocre pittore nel più spietato dittatore del Novecento: Adolf Hitler), La regina stregata di Versailles (ambientata nell'epoca di Maria Antonietta), Il Diavolo di Little Big Horn (che fornisce una versione alternativa, "demoniaca" della famosa battaglia in cui gli indiani inflissero una sconfitta leggendaria al generale Custer).

Un quinto livello riguarda il diretto riferimento a personaggi, simboli o topoi della Commedia. In una delle iniziali vignette di Devilman, Ryo per avvalorare la sua tesi dell'esistenza dei demoni, cita proprio la Divina Commedia, quale esempio di viaggio realmente compiuto negli inferi (Fig. 7). In Mao Dante e Shin Mao Dante si segnala il rinvio ad alcuni dei castighi riservati ai peccatori: i corpi di alcuni dei terrestri sono consumati dal fuoco, si riformano e sono nuovamente condannati a bruciare, in un loop ininterrotto; nella punizione denominata "inferno dello scorticamento" altri umani sono scuoiati e spellati ancora vivi; infine, nell' "inferno della dispersione" gli esseri viventi sono tagliati a pezzi e condannati a vivere ancora in quella condizione (Figg. 8a-8b-8c). In Devilman, in un frangente della storia, ad Akira/ Devilman viene annunciato che sarà guidato nelle profondità della Terra da Agwel, messaggero degli Inferi, la cui figura non può che rinviare al Caronte dantesco (Fig. 9). Un altro riferimento può essere colto nel decisivo capitolo che precede la conclusione della saga, Il tracollo, nell'episodio della morte di Miki, la ragazza, coinquilina e innamorata di Akira, che viene barbaramente decapitata, insieme al fratellino, da un'orda di umani decisi a liberare la loro abitazione dal Demonio, proprio a causa dello svelamento della vera identità demoniaca di Akira. Il senso di colpa di Akira/Devilman è raffigurato da Gō Nagai in una splendida splash page in cui il corpo evanescente, etereo di Miki si squarcia orribilmente (Fig. IOa). È solo di fronte all'estremo sacrificio della giovane che Devilman prende consapevolezza dei sentimenti nutriti per Miki, sublimati nella sepoltura del suo capo (Fig. Iob), in un rito che, dalla croce 
apposta in cima alla collina, si direbbe cristiano. Questa sequenza conduce alla definitiva scelta di campo di Akira, che smaschera i disegni di morte e distruzione dell'amico Ryo/Satana, ma, soprattutto, istituisce una sorta di virtualizzazione e sublimazione del rapporto amoroso con Miki, che, agli occhi dei più attenti lettori dell'opera omnia di Devilman, può essere interpretato come un inequivocabile, per quanto tenue, rinvio alla relazione tra Dante e Beatrice. ${ }^{\mathrm{I}}$ Sempre nella prospettiva di una relazione diretta con il testo dantesco, possiamo interpretare, in Mao Dante e Shin Mao Dante, la particolare collocazione del Re Demone Dante tra i ghiacci, che appare evidentemente una soluzione mutuata dal Lucifero della Commedia (Inf. XXXIV). Tale prelievo dall'Inferno dantesco, d'altronde, viene reiterato in una vignetta di Devilman, in cui Ryo spiega ad Akira che Satana è stato rinchiuso, per volontà divina, in un "mondo di ghiaccio". Nel citato penultimo capitolo di Devilman, inoltre, durante una conversazione, Akira/Devilman comprende che Satana è in realtà un angelo caduto - elemento questo risolutivo per smascherare le vere sembianze di Ryo (Fig. Io): in questo caso, la storia gonagaiana è così strettamente debitrice al Lucifero della tradizione cristiana, mediato ovviamente dalla Commedia dantesca, da non necessitare di ulteriori riflessioni. A fugare ogni dubbio, infine, ci sono le parole dello stesso Gō Nagai in un'intervista di alcuni anni fa (Prisco 2007), in cui, a chi gli chiedeva quali fossero i personaggi della Commedia più simpatici, così rispondeva: "Sicuramente Lucifero. Una creatura immensa, bloccata tra i ghiacci al centro della terra. Da lì partii alla fine degli anni Sessanta per realizzare Mao Dante, una storia horror dichiaratamente ispirata alla Divina Commedia. L'opera restò incompiuta, ma da Mao Dante qualche anno più tardi è nato Devilman, uno dei personaggi a cui tengo di più".

Un sesto e ultimo livello riguarda gli omaggi visivi alla traduzione figurativa di Doré. In Mao Dante l'aspetto del Re Demone Dante - irsuto e dotato di enormi ali di pipistrello - ricorda quello dei diavoli delle Malebolge. Il caso più evidente concerne l'ammasso di corpi di viventi umani, con cui il Dio alieno fabbrica i guerrieri giganti Adam ed Eve in Shin Mao Dante, che ricorda diverse configurazioni di dannati nell'Inferno raffigurato da Doré (Fig. II). Gli stessi diavoli di Malebolge, ancora in Shin Mao Dante, fungono da modello iconografico per la creatura frutto della fusione tra i gemelli Satan e Lucifer, uniti per i fianchi come gemelli siamesi, che, dai diavoli, mutuano caratteristiche fisiche come le corna, le ali, il ghigno. Ancora in Shin Mao Dante, infine, Dante si avvale di scienziati (che portano nomi di demoni della tradizione cristiana), con i quali mette a punto un sistema tecnologicamente avanzato che consente loro, assorbendo l'energia del Dio invasore spaziale, di fondersi con i propri robot avanzati e diventare demoni guerrieri. Uno di questi scienziati,

I. Ringrazio Gino Frezza per l'illuminante conversazione in proposito. 
Asmodeo, nella sua forma demoniaca, ha le sembianze di una bestia con tre facce da cane, evidentemente ricollegabile alla figura di Cerbero.

\section{Dante Shinkyoku:}

\section{SCELTE ESTETICHE E FLUSSI INTERCULTURALI}

Come abbiamo provato ad evidenziare, sia in Mao Dante che in Devilman, l'impronta dell'immaginario e della poetica danteschi informano a sprazzi il lavoro di Gō Nagai. Tuttavia per il mangaka nipponico la Commedia dantesca continua ad essere un'opera con la cui grandezza non smette mai di misurarsi. Quando egli scrive e disegna Dante Shinkyoku (tre volumi pubblicati tra il I994 e il I995), è naturale che il suo lavoro sia dunque il risultato di questa lunga ricerca.

Rispetto ai vari omaggi, citazioni, richiami, più o meno diretti, di cui sono disseminate le opere precedenti, con l'adattamento a fumetti della Commedia Gō Nagai si mostra più fedele alla fonte dantesca, a partire da due elementi. In primo luogo, il fumettista nipponico ha finalmente l'occasione di relazionarsi con quel patrimonio iconografico universale, costituito dalle illustrazioni di Gustave Doré (Amendola, Tirino 20I6). Ma di questo aspetto ci occuperemo nel prossimo paragrafo. In secondo luogo, l'autore giapponese ha l'ardua missione di compiere una serie di scelte estetiche indispensabili, per ridurre in immagini il corpus letterario dantesco.

L'obiettivo primario di Gō Nagai è rendere accessibile l'opera dantesca ad un pubblico orientale, il più vasto possibile. Rientrano in quest'ottica molte scelte estetiche, come quella di scrivere dialoghi assai affini a quelli originali e di privilegiare, tra le tre cantiche, l'Inferno rispetto al Purgatorio e al Paradiso. Dei tre volumi di Dante Shinkyoku, infatti, ben due sono riservati alle vicende infernali, mentre le altre due cantiche sono concentrate in uno solo. La ragione sta nel fatto che per Gō Nagai l'Inferno conserva un potenziale iconopoietico e simbolico superiore rispetto a Purgatorio e Paradiso, nei quali invece abbondano conversazioni, disquisizioni e dilemmi di ordine teologico-filosofico.

La Commedia di Gō Nagai prende il via dallo smarrimento del Sommo Poeta nella selva oscura di Firenze, città resa riconoscibile grazie a una vignetta in cui campeggia la cupola del Brunelleschi (completata nel I436, più di un secolo dopo la prima edizione della Commedia, del I32I), ovvero grazie a un anacronismo, che comunque è perfettamente in linea con il sincretismo dell'universo manga di cui abbiamo scritto precedentemente. Il terrore di Dante all'apparizione nella selva delle tre bestie (la lonza, il leone e la lupa, identificati come simboli della superbia, invidia e avarizia, e secondo altri metafore per Firenze, la Casa di Francia e il Vaticano) evidenzia la volontà di Gō Nagai 
di incorporare nella struttura del disegno sequenziale anche la dimensione allegorica del poema dantesco. L'arrivo di Virgilio, con l'avverarsi della profezia del veltro, segna per Dante l'annuncio del viaggio nell'aldilà. Qui va registrato un primo significativo scarto del testo gonagaiano rispetto alla fonte: mentre nel manga il Sommo Poeta si rivolge alle Muse per chiedere di saper serbare il ricordo dell'esperienza che si appresta a compiere e di saperlo trasformare in poesia, nella Commedia questa invocazione viene innalzata ex post. A nostro avviso questa variazione si inserisce in un'ottica più complessiva, già formulato dal mangaka nipponico, quella di voler accentuare le paure e l'angoscia dell'uomo Dante nell'affrontare l'immane impresa. La successiva rivelazione di Virgilio, il quale comunica a Dante che il suo viaggio è stato direttamente voluto dal Cielo su richiesta di Beatrice, innesca una lunga digressione sulla storia d'amore tra il Poeta e la donna, scomparsa a soli 28 anni. Come vedremo meglio nel prossimo paragrafo, il manga qui è costretto a deviare continuamente dal filo della narrazione per fornire al lettore giapponese gli strumenti indispensabili a contestualizzare e quindi a comprendere le vicende dell'Inferno dantesco. La raffigurazione di Beatrice segna, tuttavia, un significativo scarto rispetto alla tradizione figurativa occidentale. Gō Nagai, infatti, disegna una serie di tavole con Beatrice nuda, "avvolta da un'aura eterea ma insieme carica di una rotonda sensualità" (Tomasi 20I6) (Fig. I2), che, da un lato, riducono la storia d'amore con Dante dentro i canoni rappresentativi della bellezza nel fumetto giapponese e, dall'altro, rimarcano l'aspetto terreno del sentimento d'amore nutrito dal Poeta per la splendida fanciulla.

Come abbiamo argomentato in precedenza, il rapporto tra i manga e i classici della tradizione letteraria occidentale è incentrato sulla traduzione interculturale, finalizzata a "normalizzare" elementi culturali estranei dentro le logiche narrative e le retoriche iconiche del fumetto giapponese. Dante Shinkyoku rientra perfettamente in questa prospettiva. Nel terzo capitolo c'è un altro momento che illustra molto bene questa ricerca: è la scena, raffigurata in ogni dettaglio in cui Virgilio prende per mano Dante per sostenerlo nel passaggio attraverso l'Antinferno. Al mangaka preme sottolineare la connessione che si sta stabilendo tra i due poeti. Sempre in questa prospettiva, possiamo leggere la violenza, quasi splatter, con cui il fumetto visualizza le pene dei dannati dell'Inferno, a partire dal tormento delle mosche inflitto agli ignavi, per arrivare alla crudezza delle scene nelle Malebolge (Figg. Iza e izb). D'ora in poi il materiale letterario di partenza viene declinato in immagini plastiche ad alta dinamicità e le sofferenze dei peccatori sono esasperate dal ricorso a ogni tipo di offesa fisica.

Il passaggio attraverso il cerchio dei non battezzati, che serve per bypassare le complesse implicazioni morali e teologiche del Limbo, funge anche da preludio all'approdo al secondo cerchio, popolato dalle anime dei lussuriosi. 
Se dal punto di vista narrativo questo capitolo di Dante Shinkyoku è piuttosto fedele alla fonte, non mancano tuttavia alcuni scarti significativi: in primo luogo l'inquietante figura di Minosse che cinge la coda attorno al peccatore sottoposto al suo giudizio invece che attorno a sé (Fig. I4). Tale variazione s'inquadra perfettamente nella relazione quasi imprescindibile che i personaggi manga stabiliscono con il monstrum, inteso come creatura proveniente da un'altra dimensione (elemento su cui torneremo più avanti). Ma il cerchio dei lussuriosi viene ricordato soprattutto per la vicenda tragica di Paolo e Francesca, illustrata anche qui con un excursus, in accordo con l'originale. La rimediazione gonagaiana della vicenda si può cogliere soprattutto nell'accentuazione della sensualità del corpo di Francesca e nel turbamento che la sorte degli amanti provoca in Dante, disegnato con i mezzi tipici del manga (espressione sofferente, goccioline di sudore) (Fig. I5). In seguito alla visione dei due, Dante sviene e, in sogno, gli appare Beatrice, che si immerge, nuda, in una fonte. Questo scarto estetico, rispetto all'originale, serve all'autore per un doppio scopo: per un verso, sottolineare l'amore terreno provato dal poeta per Beatrice; per l'altro, focalizzare l'attenzione del lettore sul ruolo decisivo svolto dalla donna nel viaggio nell'aldilà. In ogni caso, la figura di Beatrice, candida e attraente, chiarisce a Dante che l'amore carnale, l'eros, può degenerare e far allontanare dalla retta via dell'amore puro.

Un'altra variazione figurativa si materializza nel terzo cerchio, in cui sono puniti i golosi: i peccatori sono parte del fango melmoso del girone (e non semplicemente immersi in esso come nella fonte dantesca) e in più vengono squartati e scuoiati in continuazione dal mostruoso Cerbero dalle tre teste (Fig. I6). Ci sembra interessante qui notare come la rimediazione interculturale sia addirittura antecedente alla stessa fonte originale. Cerbero, infatti, è una creatura dell'Averno che Enea incontra nel libro VI dell'Eneide e dalla descrizione virgiliana Dante mutua evidentemente la sua bestia infernale. Tuttavia già nella Commedia Cerbero ha tratti più marcati, sia per la commistione tra umano e animale, sia perché si placa solo quando Virgilio lancia in una delle sue bocche una manciata di fango, mentre nel poema classico ad Enea basta una focaccia impastata con miele e farina "magica" per acquietarlo. Infine nel manga gonagaiano c'è un'ulteriore rimodulazione: Dante atterrito lancia tra le fauci di Cerbero la testa di un dannato che si trasforma nuovamente in fango, per effetto appunto della fusione tra melma e golosi che abbiamo evidenziato più sopra (Fig. 17). Anche in questo caso a Gō Nagai preme sottolineare le condizioni estreme dell'esperienza dantesca.

Il cerchio degli avari e dei prodighi offre l'occasione di studiare un curioso caso di traduzione interculturale e intermediale. La deviazione riguarda qui la pena cui sono sottoposti questi peccatori. Nella Commedia (Inf. VII), infatti, avari e prodighi sono costretti a trascinare incessantemente enormi 
massi, mentre in Dante Shinkyoku sono condannati a spingere sacchi di monete (Fig. I8), esattamente come ne L'Inferno di Paperino (1987), storia scritta e disegnata da Giulio Chierchini e apparsa sul numero I654 di "Topolino". La finalità dell'operazione è simile, anche se in contesti estremamente diversi come l'Italia e il Giappone: in entrambi i casi, infatti, la scelta del danaro come contenuto dei sacchi serve a rendere in immagini dirette e immediate la legge morale del contrappasso per pubblici (giovanissimi italiani per la storia di Chierchini, lettori adulti giapponesi per quella di Gō Nagai) che probabilmente non hanno ancora studiato la Commedia dantesca.

Nei cerchi successivi dell'Inferno, Gō Nagai arricchisce il testo di partenza con scene spettacolari, di combattimento e di azione, come per il passaggio attraverso lo Stige, lo scontro alle porte di Dite e l'avvento del Messo celeste, senza però discostarsi in maniera evidente dalla fonte letteraria. Nell'ottavo cerchio, pur seguendo alla lettera il tracciato dantesco, Gō Nagai opera però una selezione degli incontri che i due poeti hanno con i dannati, soffermandosi solo sui più celebri, come Bertran de Born, la cui posa - si dimena con la propria testa stretta in una mano a mo' di lanterna - costituisce un riferimento icastico troppo forte per non essere ripreso nell'universo manga gonagaiano. Nel corso dell'attraversamento di questo cerchio, in diverse scene Dante e Virgilio conversano sulla natura del peccato e sull'essenza del viaggio voluto da Dio per la redenzione.

Nel settimo cerchio, presidiato dal Minotauro, degna di nota appare la scelta estetica di rappresentare i violenti uccisori come una sorta di zombie, il cui corpo si decompone perdendo brandelli di pelle e bulbi oculari, mentre sono immersi nel sangue che essi stessi hanno versato (Fig. 19a). Simile è la rappresentazione di alchimisti e falsari nell'ottavo cerchio o delle Malebolge (Fig. I9b). Sempre nell'ottavo cerchio, Gō Nagai concepisce una sconvolgente trasformazione di un dannato in serpente e la riesce a rendere con vividezza di particolari e con un'attenzione estrema alle pose plastiche dell'essere metà uomo-metà animale. Questi due esempi confermano come le retoriche visive dell'horror, mediate dalla sensibilità manga, costituiscano altre risorse per ri-mediare la materia dantesca in un linguaggio vicino al pubblico contemporaneo.

Nella stessa ottica che inquadra il rapporto con il mostruoso all'interno dei codici culturali propri di un pubblico di lettori orientali, va notato come, nelle tavole riservate alle Malebolge, il mangaka tratteggi diversi diavoli, ciascuno con il proprio carattere e la propria personalità in accordo con i suoi precedenti lavori, in particolare Mao Dante e Devilman, in cui i demoni sono raccontati e raffigurati non come una massa indifferenziata, ma come un insieme di creature dotate di caratteristiche e proprietà uniche. 
Nel nono cerchio, in cui albergano i traditori, si riscontra una differenza tra la tradizione di Doré e il disegno di Gō Nagai. Mentre, infatti, il primo osserva un regime di proporzioni tra i corpi dei giganti e quello dei "pellegrini” piuttosto credibile, Gō Nagai opta per un'estrema radicalizzazione delle differenze di altezza tra i poeti e le immense creature (Fig. 20). Una simile soluzione accentua, in forme altamente spettacolari, la fragilità dei due poeti al cospetto delle gigantesche creature infernali, ma risponde - ancora una volta - al tentativo di rendere familiare il bestiario dantesco per un lettore di manga abituato al proliferare di esseri dalle dimensioni spropositate, con cui, peraltro, quasi per intero si misura l'opera gonagaiana (dai demoni delle prime opere alla robofilia delle produzioni mecha).

Rispetto ai successivi incontri, il fumetto nipponico riserva particolare attenzione alla vicenda di Ugolino, cui è dedicato ampio spazio prima di giungere all'incontro con Satana, disegnato, però, quasi a sorpresa, in forme meno terrorizzanti al confronto di figure come Caronte, Cerbero, Minosse, Pluto e i diavoli delle Malebranche.

L'incontro con demoni e altre creature infernali nel fumetto giapponese va letto nel quadro dei codici culturali tipici del manga. Il mostruoso, identificato con forme sconcertanti e perturbanti, conserva infatti sempre una stretta connessione con la vita quotidiana e l'esperienza ordinaria. In questa prospettiva, allora, "l'incontro con l'altro da sé, mostruoso e deforme, ma in qualche modo percepito come familiare, una sorta di moderno perturbante sembra essere la chiave di lettura privilegiata" (Tomasi 2016). In questa prospettiva, possiamo inquadrare meglio il rapporto tra Dante e i peccatori, che, come vedremo a breve, si riproporrà anche nel Purgatorio. Nella raffigurazione che ne offre Gō Nagai, il Sommo Poeta vive quasi fisicamente le pene dei dannati: "non solo perde i sensi come nella fonte, ma prova una perenne e visibile sofferenza: suda, ansima, non sopporta il fetore che emana dai cerchi infernali" (Bombara 20I8: 128). Come loro, deve sfuggire la ferocia delle bestie infernali. In proposito, appare emblematico l'episodio di Caronte: quando il nocchiere infernale colpisce barbaramente una donna per farla salire sulla sua barca, Dante sviene e Caronte solleva anche lui, in quanto da svenuto è "come un morto" (Fig. 2I): "le due immagini dei corpi sospesi al braccio di Caronte sono quasi identiche: Dante, svenuto e quasi morto, entra nell'inferno come un qualsiasi dannato. Egli è peccatore fra i peccatori” (Bombara 20ı8: I27). Provando empaticamente il dolore dei dannati, Dante sembra sperimentare sul suo stesso corpo che la linea di demarcazione tra Bene e Male (di cui si è detto nel terzo paragrafo a proposito delle altre opere gonagaiane) è piuttosto sottile e pertiene alla necessità di compiere delle scelte etiche ardue di fronte alle complicate circostanze della vita. Emblematico, in questo senso, appare l'incontro con il suo maestro Brunetto Latini, finito nel girone dei sodomiti 
(Fig. 22). Solo raramente il Sommo Poeta si rivolge con durezza alle anime dannate, come accade nei riguardi di Filippo Argenti o dei sacerdoti corrotti (Figg. 23a-23b). La sua tensione morale raggiunge l'apice quando, poco prima di abbandonare gli inferi, si rivolge a Beatrice, con il volto rigato dalle lacrime, per confermare che terrà fede alla sua missione di narrare ai viventi le pene infernali, pur non sentendosene all'altezza (Fig. 24).

Con l'uscita dall'Inferno si chiude il secondo volume di Dante Shinkyoku. A differenza della fonte letteraria, dove all'uscita dalla burella (il tunnel tra le rocce che segna il passaggio tra i due mondi) Dante e Virgilio sono abbagliati dalla luce dell'angelo nocchiero (Purg. II), nel manga si staglia l'abbagliante apparizione di Beatrice - da leggersi, come scritto in precedenza, nel quadro di una precisa scelta estetico-narrativa dell'autore nipponico di sottolineare la centralità della donna nel viaggio ultraterreno.

Il terzo volume della trilogia comincia quindi con l'incontro con Catone che invita Dante a sottoporsi a un rito purificatorio prima di iniziare il suo cammino attraverso il Purgatorio. Il fumettista deve nuovamente affrontare la questione della selezione delle vicende da raffigurare e propende per una massiccia riduzione degli incontri, focalizzando energie e tavole sul personale percorso di redenzione del Poeta. In questa chiave l'incontro con Casella (l'artista che aveva musicato alcune delle canzoni di Dante) ricollega il viaggio ultramondano del Poeta alla sua vita terrena. Nell'attraversamento del Purgatorio, Dante è segnato, sulla fronte, da sette "P" (una per ciascun peccato capitale) (Fig. 25), che vengono cancellate ad ogni passaggio tra le varie anime colpevoli. L'intera sezione riservata da Gō Nagai al Purgatorio è costruita su una trama di tavole e vignette tutte focalizzate sui limiti e sulle paure di Dante, che in più casi prova un genuino sentimento di empatia verso le pene delle anime in attesa di ascendere al Paradiso (Belacqua, Bonconte di Monfeltro, Pia de' Tolomei, Sordello, Marco Lombardo). Questo sentimento raggiunge il suo acme quando Dante incrocia i superbi, del cui peccato egli crede di essersi macchiato più volte in vita, essendosi presentato spesso come un grande poeta. L'immedesimazione del Sommo Poeta nelle sofferenze dei superbi è resa in maniera visivamente eccellente da Gō Nagai attraverso l'adozione, da parte di Dante, della stessa postura delle anime (curvate sotto l'enorme peso che sono condannate a trasportare) (Fig. 26). Un altro momento ad alta intensità emotiva è quando Dante si accorge che Virgilio non ha potuto seguirlo in Paradiso. Gō Nagai riesce a renderne mirabilmente il pathos passando da uno stile armonioso a quello ruvido e nervoso della splash page a doppia pagina in cui è contenuto il mezzobusto del Poeta urlante (Fig. 27).

Giunti a questo punto, occorre comprendere quali codici culturali sono coinvolti dal rapporto con la sofferenza che il fumetto ha sinora messo in gioco. Come scrive Bombara (2018: 129), "la struttura dell'Inferno giapponese, 
e l'ordine teologico che lo informa deve essere riscoperto tramite l'esperienza di un dolore che assume funzione conoscitiva. (...) Il viaggio induce Dante a dubitare di se stesso anche perché si trova immerso in una realtà in continua trasformazione, che deve molto all'immaginario buddista". È attraverso quella sofferenza, di cui empaticamente il Poeta fa esperienza tanto nell'Inferno quanto nel Purgatorio, che può arrivare a comprendere il vero senso della colpa e della redenzione. Tuttavia, come abbiamo cercato di sottolineare in questo intero paragrafo, una tradizione religiosa e culturale estranea, come il Cristianesimo, deve essere tradotta in termini visivi tali da mediare il suo messaggio con la filosofia buddista. Proprio in questa direzione si muove il manga di Gō Nagai, nel quale particolare enfasi è posta sulla ciclicità delle pene: "i golosi squartati da Cerbero sono espulsi dal suo corpo sotto forma di melma, per poi riformarsi in altri corpi ed essere nuovamente divorati. (...) Inoltre, nel manga la trasformazione dei dannati occupa molte tavole ed è enfatizzata dallo sguardo disgustato del protagonista. Rientra sempre nel pensiero buddista l'interpretazione prevalente del peccato come hybris, rottura dell'equilibrio dell'universo" (Bombara 20I8: I29). In altri termini, Dante nel manga gonagaiano è immerso in un universo in perenne metamorfosi, che lo porta a dubitare delle sue stesse certezze e a interrogarsi sulla fragilità dei destini umani (Fig. 28).

Il limitato spazio riservato nel manga al Paradiso obbliga l'autore a selezionare pochi casi celebri di incontri con anime sante: Piccarda nel primo cielo, San Tommaso nel quarto, l'avo Cacciaguida nel quinto, Cristo nell'Empireo. Soprattutto ci preme sottolineare come le straordinarie tavole a doppia pagina per la rappresentazione dei diversi Cieli, in quadri finemente illustrati (Figg. 29a-29b), alimentino una nuova forma dello sguardo dello spettatore; mentre, infatti, per tutto l'Inferno e il Purgatorio la figura di Dante è sempre presente nella raffigurazione dei diversi spazi attraversati, nel Paradiso il pellegrino sparisce per lasciare spazio solo alle maestose visioni che il suo sguardo ammira.

Nelle ultime tavole dell'opera, infine, si assiste ad un progressivo espandersi di linee e tratti eterei (Fig. 30), sommersi dalla luce della beatitudine, a misura che il Sommo Poeta si approssima alla visione di Dio.

La lettura dell'intera trilogia gonagaiana fornisce all'utente mediale una significativa esperienza delle potenzialità intermediali e interculturali del fumetto, in quanto medium all'incrocio di più forme mediali e processi culturali. In altri termini, la forza delle vignette e delle tavole di Gō Nagai risiede proprio nel suo inesausto tentativo di tradurre il complesso potenziale iconopoietico dell'opera dantesca in formule visive tipiche del manga, secondo quell'approccio sincretico più volte richiamato in questo saggio. D'altro canto, in chiave mediologica, un'operazione coraggiosa come quella del mangaka giapponese è resa possibile dalle potenzialità espansive insite nel testo d'origine. Le infinite 
possibilità di sviluppo della iconopoiesi della Commedia fanno del poema dantesco un esempio di quelli che Roland Barthes (1970) definisce "testi scrivibili", ovvero testi che non solo possono essere letti, ma alimentano una produzione di ulteriori materiali nella cultura contemporanea.

\section{Mediologia di Dante Shinkyoku: TRADIZIONE FIGURATIVA (DORÉ), FORMA E RITMO DEL FUMETTO}

Come abbiamo provato ad argomentare in altra sede (Amendola, Tirino 20I6), qualsiasi traduzione visiva della Commedia dopo l'uscita dell'edizione illustrata dell'Inferno (I86I) deve necessariamente fare i conti con il lavoro di Gustave Doré. L'opera di Gō Nagai non può sottrarsi a questo destino. Infatti, è lo stesso autore a rivelare l'amore per l'illustratore alsaziano: "Ero ragazzo quando i miei fratelli portarono a casa un'edizione della Divina Commedia illustrata da Gustave Doré. Desiderai immediatamente poter disegnare come faceva lui” (Prisco 2007). Ma, prima di addentrarci nello specifico della relazione intermediale tra le illustrazioni di Doré e il fumetto di Gō Nagai, occorre riepilogare i termini della rivoluzione realizzata dall'artista francese con la creazione di un nuovo medium: il libro illustrato di grande pregio. Sono quattro i pilastri di tale rivoluzione: la ridefinizione dei rapporti tra testo scritto e illustrazione, intesa come abbandono della tecnica del disegno all'interno del testo, tecnica sostituita da immagini disegnate a tutta pagina, separate, dunque, dal testo; l'uso dell'elettrotipo, per assicurare una perfetta corrispondenza tra le incisioni e la loro riproduzione a stampa; l'adozione di un nuovo formato (in folio); la collocazione dell'illustrazione in un punto preciso del volume, protetta da una velina. Si determina così un nuovo oggetto mediale che riconfigura completamente la relazione dinamica tra scrittura, lettura e immagine, e quindi anche l'esperienza dell'utente. In pratica, Doré manipola il testo per adattarlo alla propria interpretazione visiva (Marin 2015: 15), elaborando una sorta di strategia "cliffhanger" (Cole 1994): "divide il testo in modo da suscitare la suspence del suo lettore, che doveva attendere per incontrare l'attesa immagine. Ma Doré attribuisce persino una nuova funzione alla carta velina inserita (...), usandola per dislocare e ricollocare piccole porzioni di testo: chiedeva ai tipografi di stampare sui fogli protettivi le linee di testo che si abbinavano all'illustrazione per incrementare l'intensità emotiva della fruizione spettatoriale, sovrapponendo il testo (in trasparenza) alla parte inferiore dell'immagine e, così, confondendo codice iconico e codice linguistico. La carta velina, dunque, funzionava praticamente come un sipario teatrale, che svelava l'episodio che stava per essere messo in scena ad un lettore/spettatore, intento a conferire un senso alle linee di testo appena lette sulla carta stessa" (Amendola, Tirino 20I6: 23). Siamo in presenza di una nuova qualità del rapporto tra immagine 
e testo, tra illustrazione e letteratura, che riconfigura innanzitutto il ritmo della lettura, rompendo gli schemi della figuratività classica (Abruzzese 2007) a favore dello sguardo di pubblici ormai avvezzi alle forme di audiovisione le quali preparano al salto percettivo che sarà di lì a poco garantito dall'avvento del cinematografo. Il ritmo che scandisce l'alternanza tra lettura del poema e immersione nelle immagini di Doré scaturisce da una struttura mediologica, che, con qualche necessario accorgimento, dalle edizioni illustrate del maestro francese si riverbera nel film L'Inferno (I9II), diretto da Francesco Bertolini, Giuseppe De Liguoro e Adolfo Padovan e prodotto dalla Milano Films. Nella pellicola si crea infatti "una simbiosi tra didascalia e scena, ritmata su un'estetica dell'illustrazione" (Amendola, Tirino 20I6: 23): in altri termini, nel film, che opera un vero e proprio calco iconografico dalle immagini di Doré (Bardos 20II), le didascalie - con il loro portato di commenti, spiegazioni, paratesti - determinano il ritmo di proiezione/fruizione del film, arricchendo l'esperienza spettatoriale con una mole di informazioni indispensabili per godere a pieno della narrazione filmica.

In Dante Shinkyoku assistiamo ad un'operazione altrettanto ardita. Le illustrazioni nello stile di Doré, infatti, puntellano tutta la trilogia (Figg. 3Ia-3Ib3IC-3Id-3Ie-3If-3Ig-3Ih). Ciascun passaggio importante della storia è introdotto da una vignetta (per lo più a pagina intera), che riproduce, quasi alla lettera, un'incisione di Doré, accompagnata dalla relativa terzina. Siamo in presenza di una vera e propria struttura mediale, che crea un conflitto tra due stili apparentemente agli antipodi: da un lato il tratto fitto di linee, drammaticamente calato nel conflitto tra volumi e luci, di uno stile dichiaratamente ispirato a Doré, dall'altro i mezzi audiovisivi (formule tipiche del disegno, topoi visivi per la rappresentazione della bellezza femminile, ecc.) del manga. Possiamo parlare, ancora più precisamente, di una struttura mediale matrice - quella creata da Doré nelle edizioni illustrate della Commedia - dove l'illustrazione acquisisce una funzione simbolica allo stesso tempo autonoma e in dialogo con il poema, e due strutture mediali derivate, ovvero quella del film L'Inferno, in cui didascalie e immagini in movimento vivono in simbiosi, e quella della trilogia gonagaiana, che illustreremo più in dettaglio a breve. Ciò che accomuna le tre strutture mediali è l'inserimento, in un certo continuum, di un elemento estraneo, che potenzia la narrazione e scandisce il ritmo di fruizione. Se questo elemento estraneo è in Doré evidentemente l'illustrazione a tutta pagina e nel film del ı9ı le didascalie (o intertitoli), nel manga è rinvenibile direttamente nell'inserimento di tavole che riproducono quasi alla lettera $\mathrm{i}$ disegni del maestro francese. In Dante Shinkyoku la rottura del continuum narrativo, operata con la ciclica riproduzione di alcune tavole di Doré, assolve una triplice funzione: in primis, l'inserimento della terzina del poema, anche nelle edizioni illustrate da Doré associata a quella determinata tavola, può es- 
sere letto come un devoto omaggio all'arte dell'incisore alsaziano; in secondo luogo, il puntuale uso di pagine intere nello stile di Doré detta il ritmo della lettura, poiché, lavorando come elemento ciclico che a intervalli regolari interrompe la rappresentazione figurativa del manga, determina una pausa tra una sezione e l'altra dell'opera, spesso aprendo al passaggio in una nuova zona dei regni ultraterreni; in terzo luogo, il ricorso alle tavole di Doré risponde ad una necessità di evidenziare la dimensione "esotica" del racconto per il lettore giapponese, che, entrando progressivamente in familiarità con i codici visivi del libro illustrato, si cimenta nella decodifica di un linguaggio visivo per lui inizialmente "estraneo". Per quest'ultimo punto risulta particolarmente interessante l'uso che, delle tre illustrazioni di Doré, viene fatto da Gō Nagai per raccontare la vicenda del Conte Ugolino. L'impressione è che il mangaka, ricorrendo in toto all'apparato iconografico di Doré, abbia voluto rinsaldare nel lettore la sensazione di una storia calata nell'immaginario medioevale (Fig. 32).

Il sincretismo, che, come abbiamo già visto, presiede all'incorporazione nell'universo manga di temi e figure estranee alla cultura orientale, opera, almeno in parte, anche a livello tecno-mediale. Le prime tavole prelevate dal corpus di Doré, infatti, creano uno stridore marcato con lo stile tipico del manga; ma le successive tavole inserite gradualmente si omogeneizzano con il resto del materiale iconografico manipolato da Gō Nagai a tal punto che, in alcuni casi, la narrazione procede così fluidamente da rendere difficile l'individuazione del calco. Le ultime tavole dell'opera, in questo senso, sono emblematiche. Il fumettista nipponico lascia da parte qualsiasi stilema manga, elimina quasi completamente dialoghi e didascalie e affida tutto il carico simbolico alle immagini, in cui prevalgono bagliori, accensioni e lucentezza, in marcata consonanza con le ultime incisioni di Gustave Doré. In accordo con il maestro alsaziano, Gō Nagai arresta la rappresentazione fumettistica con l'illustrazione del Trionfo dei beati nella Candida Rosa; così come si ferma di fronte all'irrappresentabile per eccellenza, ovvero l'immagine di Dio (Par. XXXIII).

In sintesi, possiamo dire che Gō Nagai lavora sull'iconografia di Doré sia per omaggiare il maestro dell'immaginario dantesco, sia per scandire la narrazione rompendo il continuum dello stile manga, sia, infine, affinché il pubblico giapponese abbia sempre presente il riferimento a una storia "esotica" di cui si deve in qualche modo saper padroneggiare i codici illustrativi, per quanto lontani dalla tradizione nipponica.

L'intenso rapporto con la tradizione iconografica di Doré non esaurisce, tuttavia, il complesso lavoro di Gō Nagai sulla forma mediale del manga Dante Shinkyoku. Il mangaka, infatti, lavora anche a produrre una serie di paratesti, in cui includere didascalie e digressioni storiche, necessarie a fornire all'ignaro pubblico nipponico una serie di informazioni storico-letterarie per 
contestualizzare le vicende raffigurate. Particolarmente rilevanti sono le parentesi in cui il manga racconta le origini dell'amore tra Dante e Beatrice, fin da quando erano bambini (Fig. 33), il rapporto amicale con il poeta stilnovista Guido Cavalcanti, il componimento della Vita Nova e le vicende biografiche della famiglia Alighieri. In pratica, "il carattere di rielaborazione dell'inferno gonagaiano viene infine potenziato dalla sua intertestualità come rete di relazioni tra la fonte, la sua 'biblioteca' e l'interpretazione attuale" (Bombara 20I8: I30). All'interno di questa tessitura intermediale, il mangaka nipponico rivela anche di conoscere molto bene opere pittoriche poco note, come il dipinto Farinata degli Uberti alla battaglia di Montaperti (I842) del pittore romantico Giuseppe Sabatelli, integralmente riprodotto in una delle vignette che narrano le vicende del capo ghibellino, confinato da Dante tra gli eretici epicurei (Fig. 34).

Un altro strumento che caratterizza la medialità del manga di Gō Nagai è l'uso dello spazio della vignetta come una sorta di schermo per la proiezione delle immagini richiamate dalle parole di uno dei personaggi: è il caso, per esempio, delle immagini dell'inferno che compaiono alle spalle di Virgilio, mentre questi illustra a Dante cosa lo attende nell'aldilà (Fig. 35); oppure del volto di Beatrice che compare nella vignetta in cui Dante le si rivolge per chiedere se veramente lui sia il prescelto per riferire al mondo gli orrori dell'Inferno. Lo stesso espediente, con il Sommo Poeta addirittura raffigurato di spalle nella vignetta mentre "guarda" i suoi ricordi, lo si ritrova nel terzo volume, quando egli ricorda alcuni dei volti distorti, deturpati e orrorifici che ha incrociato nell'Inferno.

Gō Nagai, infine, padroneggia perfettamente il linguaggio audiovisivo e conferisce alla narrazione sequenziale una fluidità eccellente, per effetto del frequente cambio di inquadrature, che, anche all'interno della stessa pagina, spaziano dal primo e primissimo piano (privilegiato per rappresentare le forti emozioni provate da Dante) ai campi lunghi e lunghissimi (per collocare, nella giusta proporzione, i due pellegrini dentro l'immensità degli spazi attraversati) (Fig. 36).

\section{Conclusioni}

La lettura che abbiamo proposto del manga giapponese Dante Shinkyoku è partita da una duplice, necessaria premessa. Da un lato, ci è parso indispensabile ribadire la forza iconopoietica della commedia dantesca che ha alimentato un numero molto ampio di opere in vari formati mediali (film, romanzi, videogames, fumetti). Dall'altro, ci è parso utile ricordare alcune tappe dell'affermazione dell'opera di Dante in Giappone, nazione in cui - soprattutto dopo le aperture dell'era Meiji - l'interesse per la Divina Commedia è rimasto 
sempre vivo, grazie alle numerose traduzioni e alle opere che, più o meno direttamente, vi si sono ispirate. In questo quadro socioculturale, si inscrive l'opera di uno tra i maggiori mangaka giapponesi, Gō Nagai, che, alla Comme$\mathrm{dia}$, ha riservato parecchie energie intellettuali. Il fumettista giapponese trova nella Commedia una miniera di stimoli, che vengono a vario titolo rielaborati e riversati in opere come Mao Dante e Devilman, entrambe cominciate all'inizio degli anni Settanta. D'altronde, il flusso interculturale attraverso il quale i fumetti di Gō Nagai attingono al patrimonio dantesco non si arresta neanche con Dante Shinkyoku, ma prosegue nel ciclo sequel di Mao Dante, chiamato Shin Mao Dante, e pubblicato dopo la Commedia gonagaiana.

Le prove e i tentativi con Mao Dante e Devilman, in cui i riferimenti sono molteplici e articolati su sei livelli (carattere dei personaggi, statuto interdimensionale, ibridazione tra bene e male, recupero dei personaggi della mitologia classica, riferimenti diretti a topoi della Commedia, omaggi alla tradizione figurativa di Doré), sono stati analizzati per rendere più chiaro quanto duraturo e stratificato nel tempo sia il rapporto dell'autore con la Commedia dantesca, soprattutto nella versione illustrata da Doré. Alla luce di queste osservazioni preliminari, nei due paragrafi successivi ci siamo concentrati sull'analisi socioculturale e mediologica di Dante Shinkyoku, avendo ben presenti le coordinate della biografia artistica dell'autore in merito al suo rapporto con Dante e Doré.

Tale analisi ha rivelato come il manga gonagaiano funzioni come esempio illuminante delle qualità del medium fumettistico. Nella tradizione del sincretismo, modalità culturale con cui i mangaka traducono elementi estranei in termini linguistici e simbolici comprensibili al pubblico giapponese, Gō Nagai compie un'ardita opera di semplificazione e divulgazione della Commedia dantesca, da un lato rispettandone, sostanzialmente, la potenza iconopoietica e drammaturgica, dall'altro adottando una serie di deviazioni e riduzioni utili a renderne più accessibile il messaggio, anche in termini iconici, a un pubblico non avvezzo alla lettura del poema.

Il lavoro del mangaka valorizza al massimo grado le qualità mediali del fumetto, grazie alla sua possibilità di generare e gestire "strati di significazioni, fra immagini e scritture, fra statico e dinamico, fra visivo e linguistico, verbale e non verbale, descrittivo e fantastico" (Frezza 2005: 47). In sintesi, si potrebbe affermare che il manga di Gō Nagai lavori come spazio di comunicazione intermediale e interculturale. Attraverso una serie di complessi procedimenti che abbiamo provato a evidenziare nelle pagine precedenti, Dante Shinkyoku manipola un patrimonio letterario e storico assai lontano dalle forme di comunicazione del Giappone contemporaneo (per quanto, come spiegato, la Commedia non sia affatto sconosciuta in territorio nipponico), trasformandolo, grazie all'immediatezza delle tavole e alla dinamica plasticità dello stile 
del disegno, in un racconto che oltrepassa vari steccati linguistici e storici. Rispetto alla libertà con cui ha rielaborato il materiale dantesco nelle opere precedenti, il mangaka mantiene qui un atteggiamento di maggiore fedeltà ad un'iconografia, quella costruita da Gustave Doré, con cui è impossibile non confrontarsi per chiunque voglia elaborare l'immaginario visivo della Commedia. Tuttavia, anche in un contesto di attenzione alla fonte iconografica dell'illustratore alsaziano, Gō Nagai progetta una macchina mediale in cui le incisioni di Doré, rompendo il continuum della narrazione sequenziale del manga, rappresentano, contestualmente, un omaggio all'artista così amato, un tentativo di ritmare il racconto e, infine, una evidenziazione della matrice "esotica", per i lettori giapponesi, della fonte letteraria originaria. Lo straordinario manga di Gō Nagai, in conclusione, attesta nuovamente le opportunità di pensare il fumetto come spazio di rimediazione (Bolter, Grusin 1999) tra forme mediali differenti e tra culture lontane, ovvero come un medium aperto ai processi di traduzione da medium a medium e da codici culturali ad altri codici culturali, assai differenti fra loro, secondo ritmi di innovazione sociale e mediale ogni volta rigenerati e imprevedibili (Berninger, Ecke, Haberkorn 20IO, Duncan, Smith 2009, 20II, Lyons, Williams 20I0, Frezza 20I7, Tirino 20I8). 


\section{BIBLIOGRAFIA}

Abruzzese, A., 2007, La Grande Scimmia, Roma, Sossella.

Amendola, A., Tirino, M., 20I6, "Il filtro di Dante. L'impronta di Gustave Doré dal cinema muto al digitale", Dante e l'arte, 3, pp. II-38.

Bandirali, A., 1999, Dal verso all'immagine. Riflessione su luoghi e natura nella Commedia dantesca: L'Inferno, Varese, Lativa.

Bardos, J., 20II, "Dante e il cinema", in Vígh, É. (a cura di), Leggere Dante oggi, Roma, Aracne.

Barthes, R., 1970, $S / Z$, Paris, Éditions du Seuil.

Benucci, A., 20I8, "Dalla Commedia ai comics: costanti e varianti di critica sociale nelle riscritture fumettistiche contemporanee dell'Inferno di Dante", in Lazzarin, S., e Dutel, J. (a cura di), Dante pop. La Divina Commedia nella letteratura e nella cultura popolare contemporanea", Manziana, Vecchiarelli, pp. IO7-I2O.

Berninger, M., Ecke, J., Haberkorn, G. (eds.), 2010, Comics as a Nexus of Cultures. Essays on the Interplay of Media, Disciplines and International Perspectives, Jefferson, McFarland \& Company.

Bouissou, J.-M., 2oro, "Manga: A Historical Overview", in Johnson-Woods (ed.), Manga: An Anthology of Global and Cultural Perspective, New York, Continuum, pp.17-33.

Bouissou, J.-M., 20II, Il manga. Storia e universi del fumetto giapponese, Latina, Tunué.

Bombara, D., 20I8, "Viaggi comic di Dante e Beatrice fra Giappone, Usa e Italia: un'inedita Commedia dolorosa, combattiva, ma anche di umanissima semplicità quotidiana", in Lazzarin, S., e Dutel, J. (a cura di), Dante pop. La Divina Commedia nella letteratura e nella cultura popolare contemporanea, Manziana, Vecchiarelli, pp. I2I-I35.

Capponcelli, L., 20I4, "Dal Paradiso all'Inferno: la Divina Commedia in Giappone attraverso Masamune Hakuchō e Akutagawa Ryūnosuke”, in Sturiale, M., e Traina, G. (a cura di), Parole e sconfinamenti, Leonforte, Euno, pp. 73-85.

Castello, M.G., Scilabra, C., 20I5, "Theoi becoming Kami. Classical mythology in the anime world", in Carlà, F., Berti, I. (eds.), Ancient Magic and the Supernatural in the Modern Visual and Performing Arts, London-New York, Bloomsbury, pp. I77-196.

Clements, J., McCarthy, H., 2006, The Anime Encyclopedia. Revises \& Expanded Edition. A Guide to Japanese Animation Since 19I7, Berkeley, Stone Bridge Press.

Cole, W., 1994, "Literal Art? A New Look at Dorés Illustrations for Dante's Inferno", Word \& Image, I0.2, pp. 95-106.

Danesi, A., 20I6, "Romics 20I6, intervista al grande Gō Nagai", Movie for Kids, disponibile all'indirizzo http://www.movieforkids.it/news/romics-2016-intervista-algrande-go-nagai/40537/ [ultimo accesso il 21.09.2018].

De Angelis, R., I967, Il colore nella Divina Commedia (nell'Inferno e nel Purgatorio), Napoli, Loffredo.

Di Fratta, G., 2007, "Robot Anime. Robofilia e tecnocentrismo nel cinema di animazione giapponese", in Di Fratta, G. (a cura di), Robot. Fenomenologia dei giganti di ferro giapponesi, Caserta, L'aperìa, pp. 43-68.

Doi, H., 20I6, "Due lezioni su Dante e Pasolini", in In continua ricerca di voci: saggi in onore del professor Tadahiko Wada, Kyoto, Eimei, pp. II-I9.

Duncan, R., Smith, M.J. (eds.), 2oII, Critical Approaches to Comics: Theories and Methods, New York-London, Routledge.

Frezza, G., I995, La macchina del mito tra film e fumetti, Firenze, La Nuova Italia.

—, 1999, Fumetti, anime del visibile, Roma, Meltemi. 
—, 2005, "Passare attraverso. Figurare, impaginare, iconizzare", in Barbieri, D. (a cura di), La linea inquieta. Emozioni e ironia nel fumetto, Roma, Meltemi, pp. 47-73.

-, 2008, Le carte del fumetto, Napoli, Liguori.

-, 2017, Nuvole mutanti, Milano, Meltemi.

Fujisawa, M., 1965, Dante nella letteratura moderna del Giappone, Annuario dell'Istituto Giapponese di Cultura di Roma, II, pp.75-89.

Fujitani, M., 200o, Shinkyoku, il canto divino. Leggere Dante in Oriente, Trento, Dipartimento di Scienze Filologiche e Storiche.

Gilson, M., 1998, "A Brief History of Japanese Robophilia", Leonardo, 3I(5), pp. 367-369.

Gō Nagai, 2006, La Divina Commedia I, Milano, d/books.

-, 2006, La Divina Commedia 2, Milano, d/books.

—, 2007, La Divina Commedia 3, Milano, d/books.

-, 20I6, Shin Mao Dante I, Milano, Edizioni BD.

-, 2017, Shin Mao Dante 2, Milano, Edizioni BD.

-, 2017, Shin Mao Dante 3, Milano, Edizioni BD.

—, 2017, Shin Mao Dante 4, Milano, Edizioni BD.

-, 2017, Devilman Omnibus, Milano, Edizioni BD.

—, 20I8, Mao Dante. Vol. I, Milano, Edizioni BD.

-, 2018, Mao Dante. Vol. 2, Milano, Edizioni BD.

-, 20I8, Mao Dante. Vol. 3, Milano, Edizioni BD.

Hofer, M., 2010, "L'Inferno": dal testo dantesco all'immagine cinematografica in Italia, Wien, Universitat Wien.

Ito, M., Okabe, D., Tsuji, I. (eds.), 20I2, Fandom Unbound: Otaku Culture in a Connected World, New Heaven-London, Yale University Press.

Iwakura T., 200I, "La fortuna di Dante in Giappone", in Boscaro, A., Bossi, M. (a cura di), Firenze, il Giappone e l'Asia Orientale, Firenze, Olschki, pp. 27I-279.

Kure, S., I965, "Dante in Giappone", Il Giappone, 5, pp. 9-I7.

Lyons, J., Williams, P. (eds.), 20I0, The Rise of the American Comics Artist. Creators and Contexts, Jackson, University Press of Mississippi.

Marin, I., 20I5, "Dante's Hell Envisioned by Gustav Doré: An Overlooked Opening to Modernity", IJCCSEC, 4(I), pp. 8-I8.

Nogami, S., I965, "Dante in Giappone", in Branca, V., e Caccia, E. (a cura di), Dante nel mondo, Firenze, Olschki, pp. 229-235.

Pellitteri, M., 2008, Il Drago e la Saetta. Modelli, strategie e identità dell'immaginario giapponese, Latina, Tunué.

Ponticiello, R., Scrivo, S. (a cura di), 2007, Con gli occhi a mandorla. Sguardi sul Giappone dei cartoon e dei fumetti, Latina, Tunué.

Prisco, F., 2007, "Gō Nagai, il padre di Goldrake: 'Devilman? È figlio del Lucifero di Dante", Il Sole 24 Ore, 27 aprile, disponibile all'indirizzo http://www.ilsole24ore. com/art/SoleOnLine4/Tempo\%2olibero\%20e\%20Cultura/2007/04/intervistanagai-prisco.shtml?refresh_ce=I [ultimo accesso il 25.06.2018].

Raffaelli, L., I993, "Dante come Mazinga”, La Repubblica, 26 gennaio, p. 37.

Scilabra, C., 20I5, "Vivono fra noi. L'uso del classico come espressione di alterità nella produzione fumettistica giapponese", Status Quaestionis, 8, pp. 92-IO9.

Tirino, M., 20I8, "Nuvole e pixel. Per una lettura sociologica del fumetto sperimentale contemporaneo", in Bifulco, L., e Santoro, A. (a cura di), Sguardi dalle scienze sociali, Napoli, Funes/Ipermedium, pp. 69-94.

Tomasi, F., 2016, "Dante reloaded: il poema dantesco e l'universo manga di Gō Nagai”, Arabeschi, VII, pp. 258-262. 

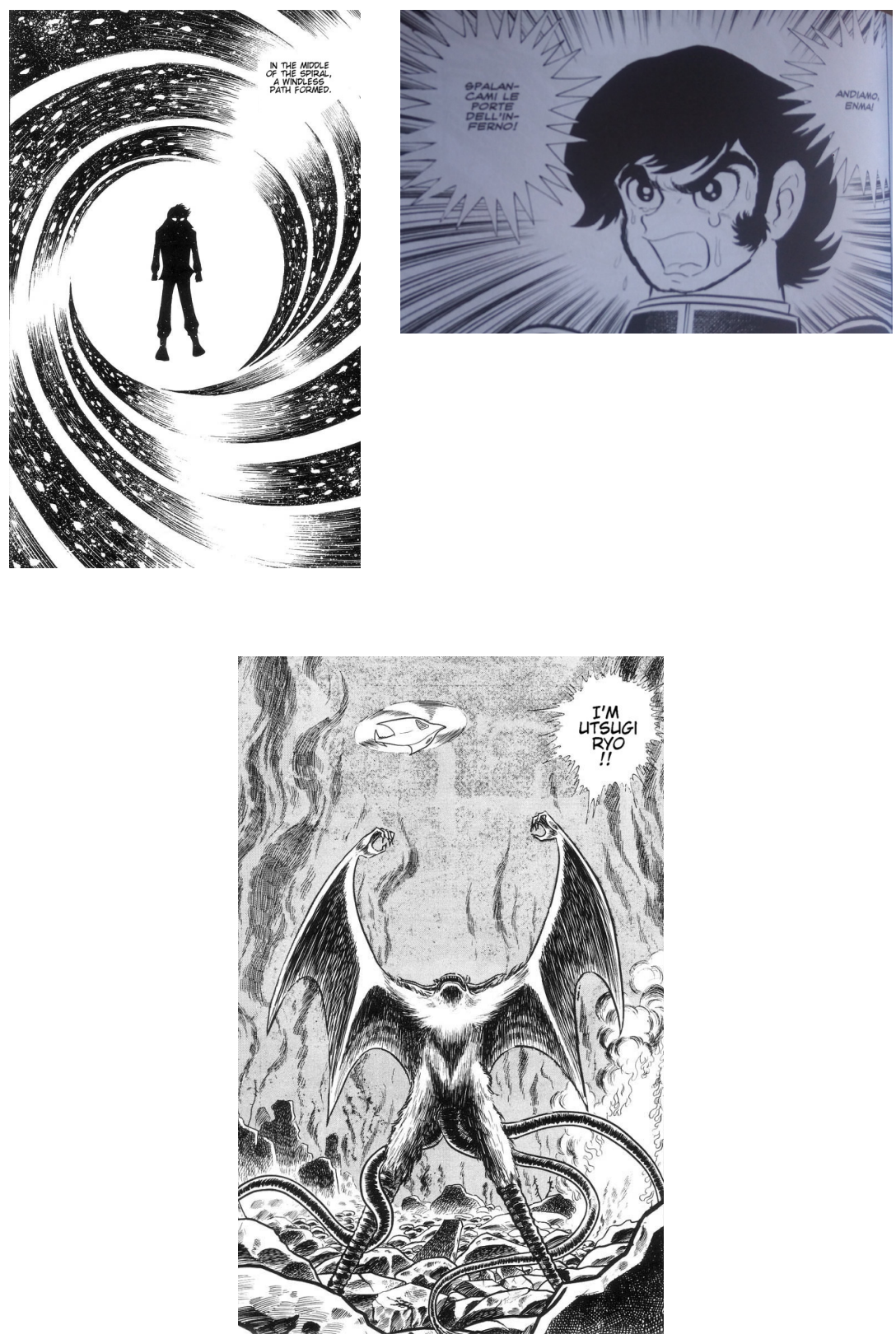

Figura Ia. Ryo Utsugi (Mao Dante, I97I)

Figura Ib. Akira Fudo (Devilman, 1972)

Figura 2. Ryo Utsugi/Re Demone Dante (Mao Dante, I97I) 

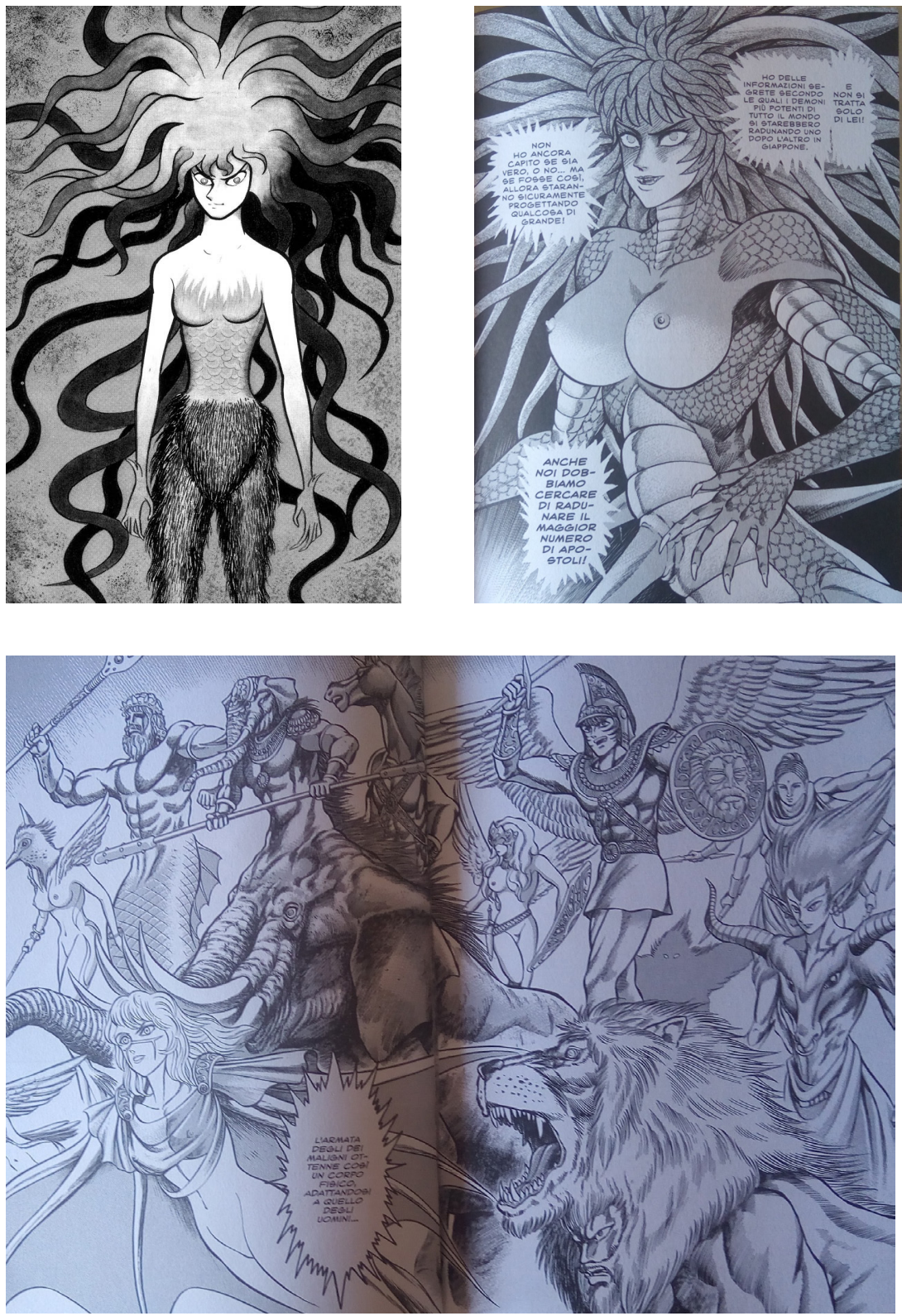

Figura 3a. Vignetta con Medusa (Mao Dante, I97I)

Figura 3b. Vignetta con Medusa "tecnologica" (Shin Mao Dante, 2002) 

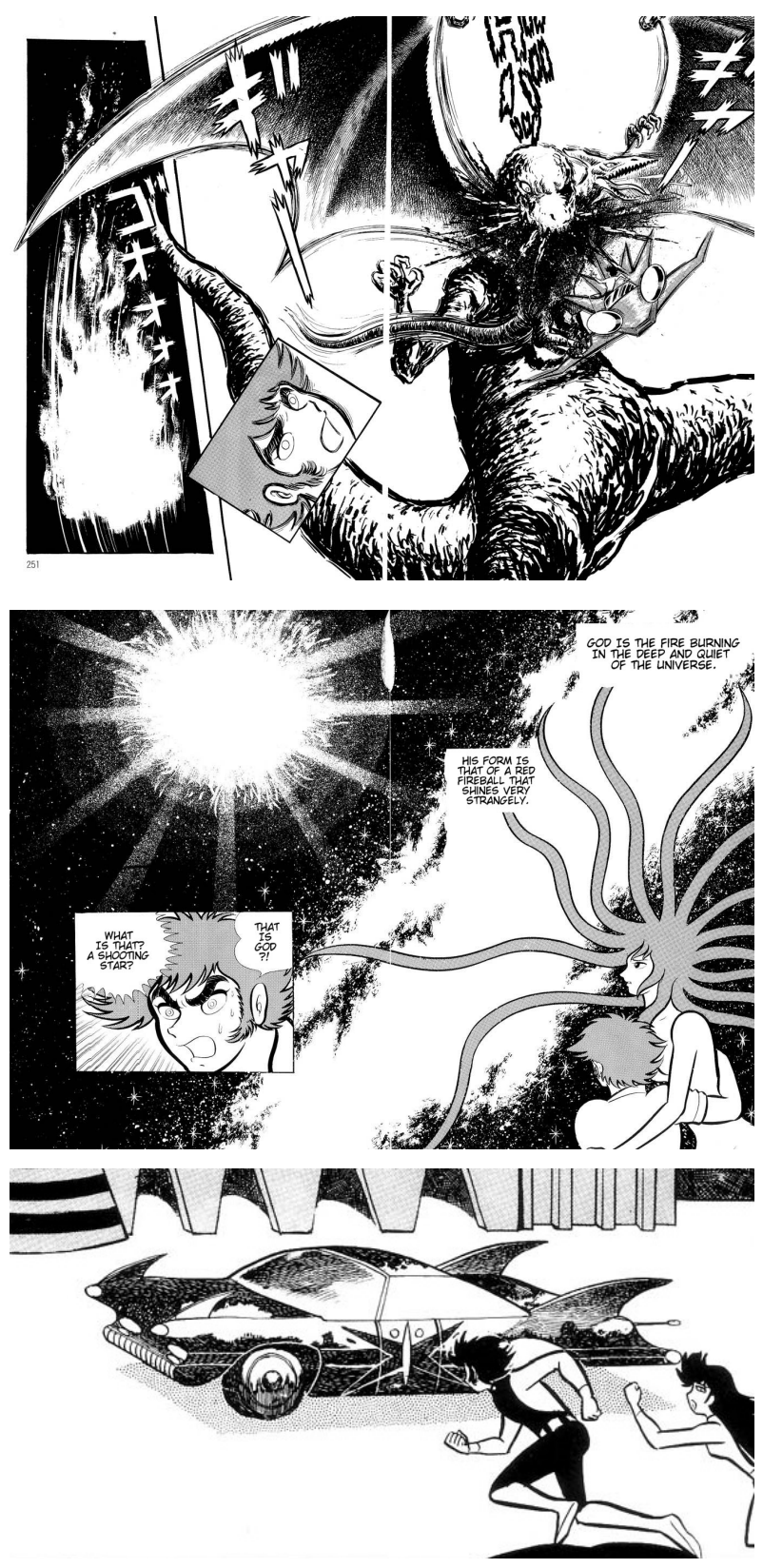

Figura 4. Pagina con diretti riferimenti all'iconografia dei kaiju eiga (Mao Dante, 197I).

Figura 5. Medusa rivela a Ryo/Dante che Dio è una massa di energia venuta dallo spazio (Mao Dante, I97I).

Figura 6. Ryo/Dante utilizza un'auto simile alla Batmobile (Mao Dante, 197I). 

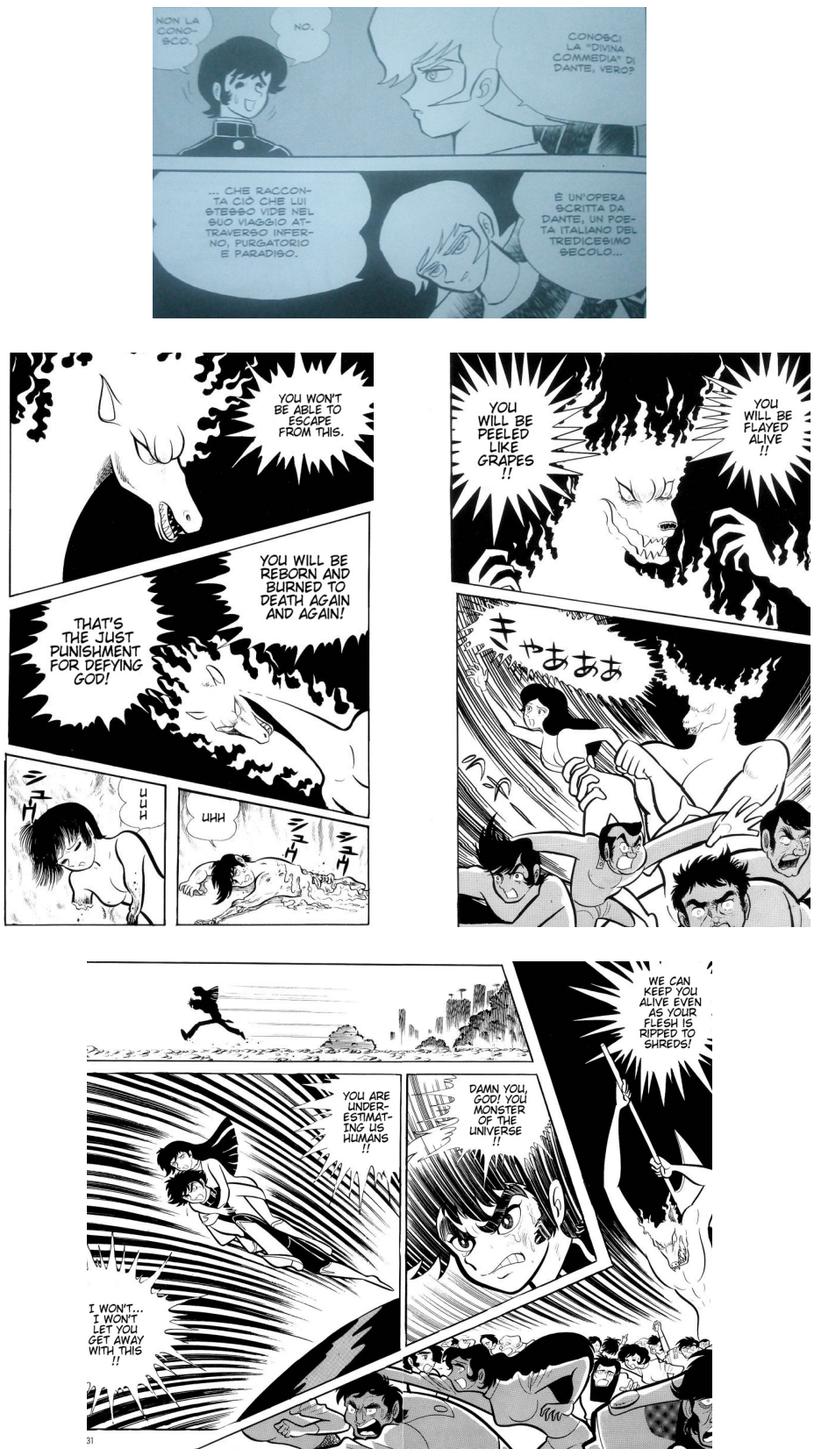

Figura 7. Ryo cita la Divina Commedia (Devilman, 1972).

Figure $8 \mathrm{a}-8 \mathrm{~b}-8 \mathrm{c}$. I castighi riservati da "Dio" invasore spaziale agli originari terrestri (Mao Dante, I97I). 

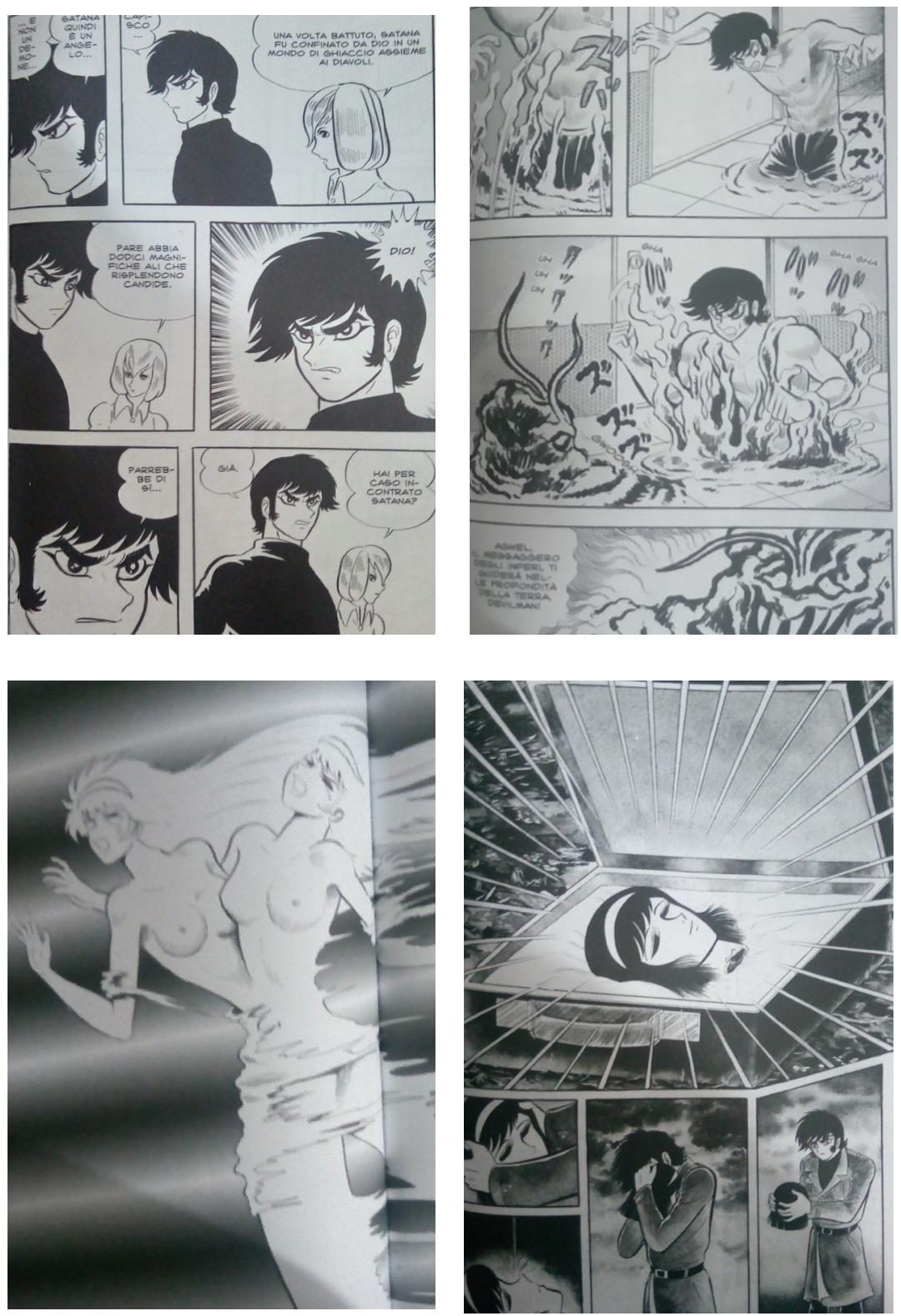

Figura 9. Akira/Devilman riceve la notizia che il messaggero degli inferi, Agwel, lo condurrà negli inferi (Devilman, 1972).

Figura Ioa. Nell'immaginazione di Akira, il corpo di Miki (appena uccisa) si squarcia orribilmente (Devilman, 1971).

Figura Iob. Akira/Devilman seppellisce la testa di Miki (Devilman, 197I). 

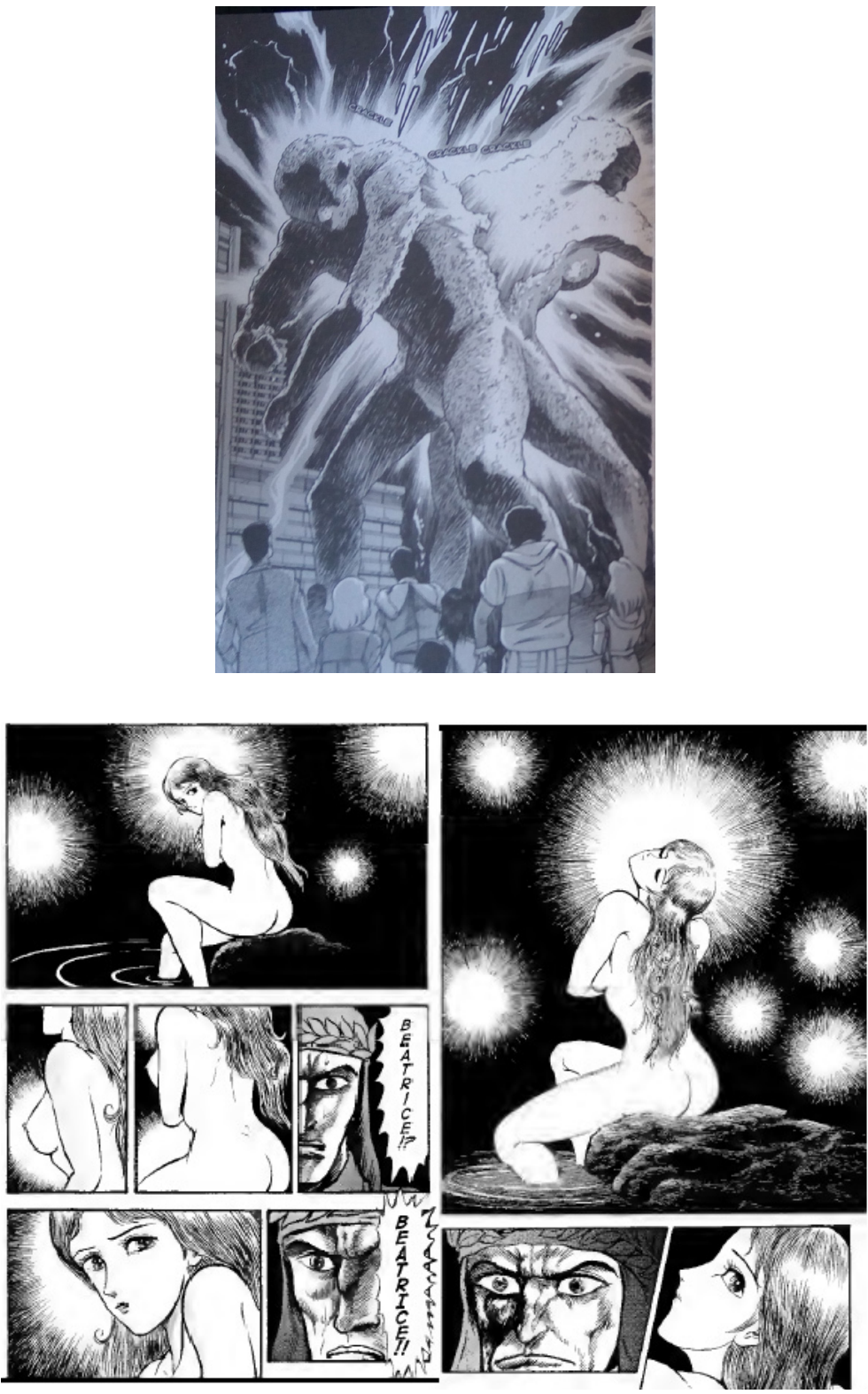

Figura II. I corpi di Adam ed Eve composti da migliaia di corpi di terrestri (Shin Mao Dante, 2002).

Figura I2. Beatrice appare in sogno a Dante (Dante Shinkyoku, 1994). 

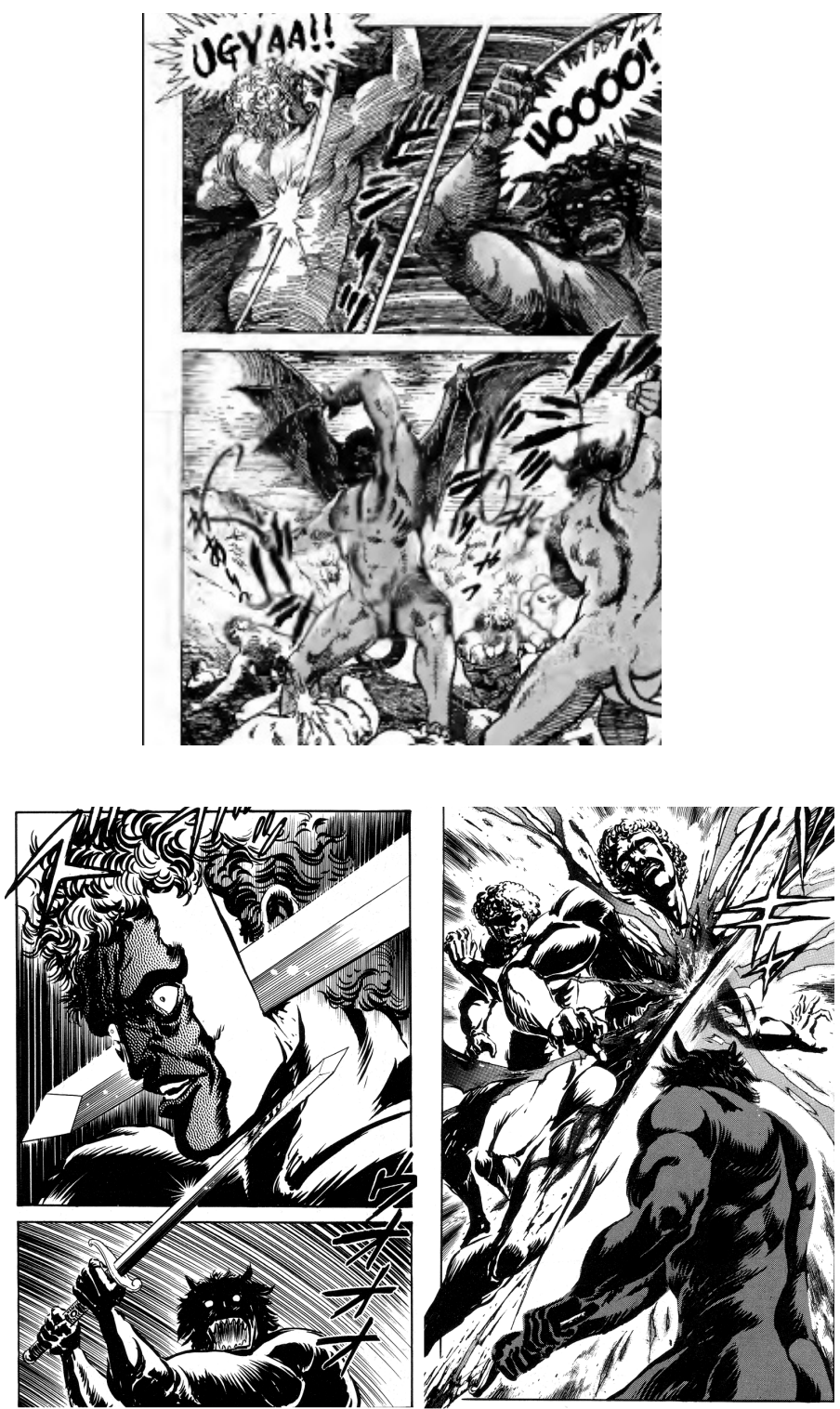

Figure I3a-I3b. Le tremende punizioni perpetrate dai diavoli delle Malebolge (Dante Shinkyoku, I994). 

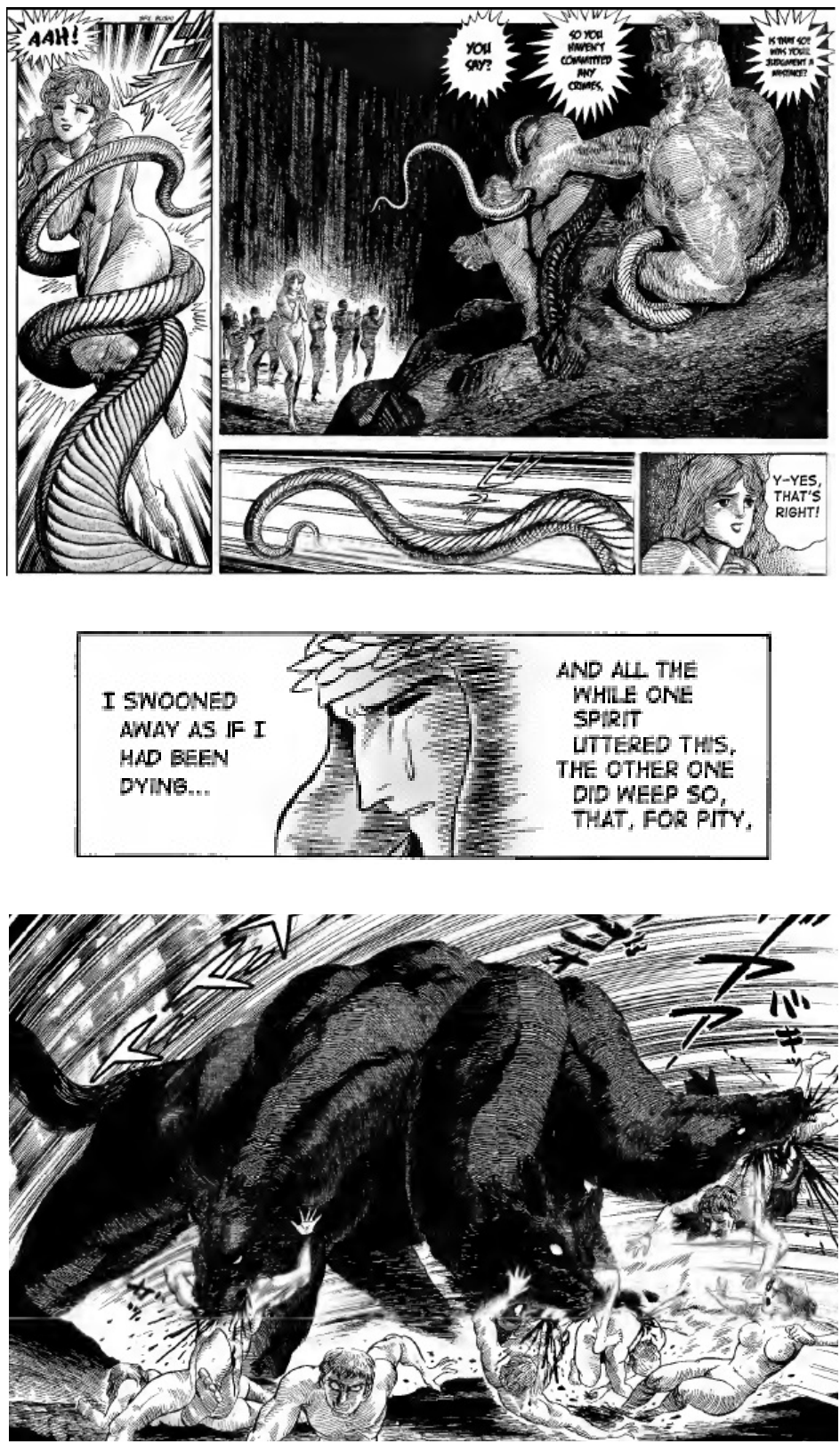

Figura I4. Minosse avvolge la coda attorno a un dannato (Dante Shinkyoku, 1994). Figura 15. Dante in pena per la sorte di Paolo e Francesca (Dante Shinkyoku, 1994). Figura I6. Cerbero squarcia le carni dei golosi (Dante Shinkyoku, 1994) 

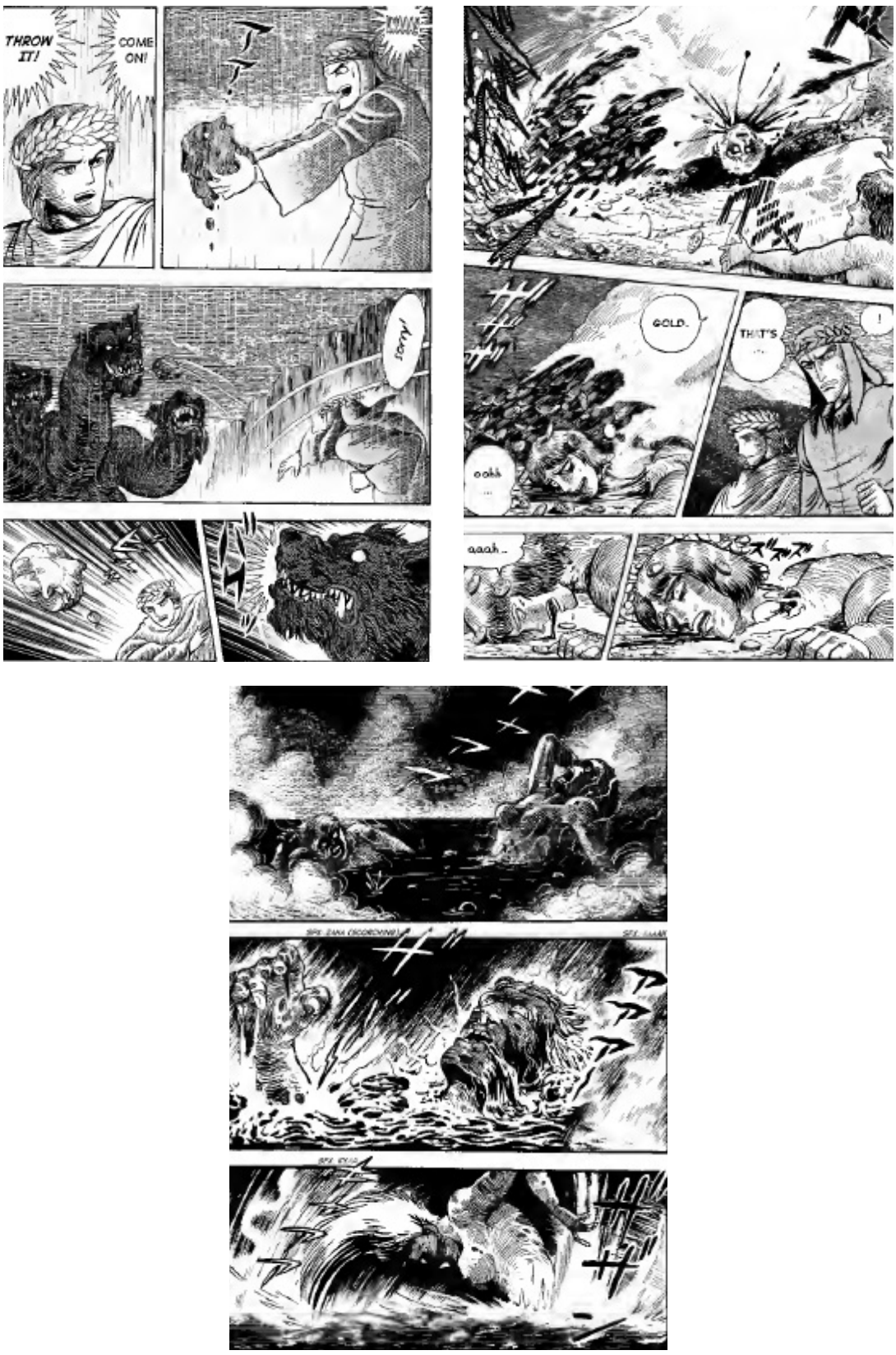

Figura 17. Dante lancia la testa di un dannato, che si tramuta in fango, in una delle bocche di Cerbero (Dante Shinkyoku, 1994).

Figura I8. Gli avari trascinano enormi sacchi di monete (Dante Shinkyoku, 1994).

Figure 19a-I9b. Dannati rappresentati come zombie (Dante Shinkyoku, 1994). 

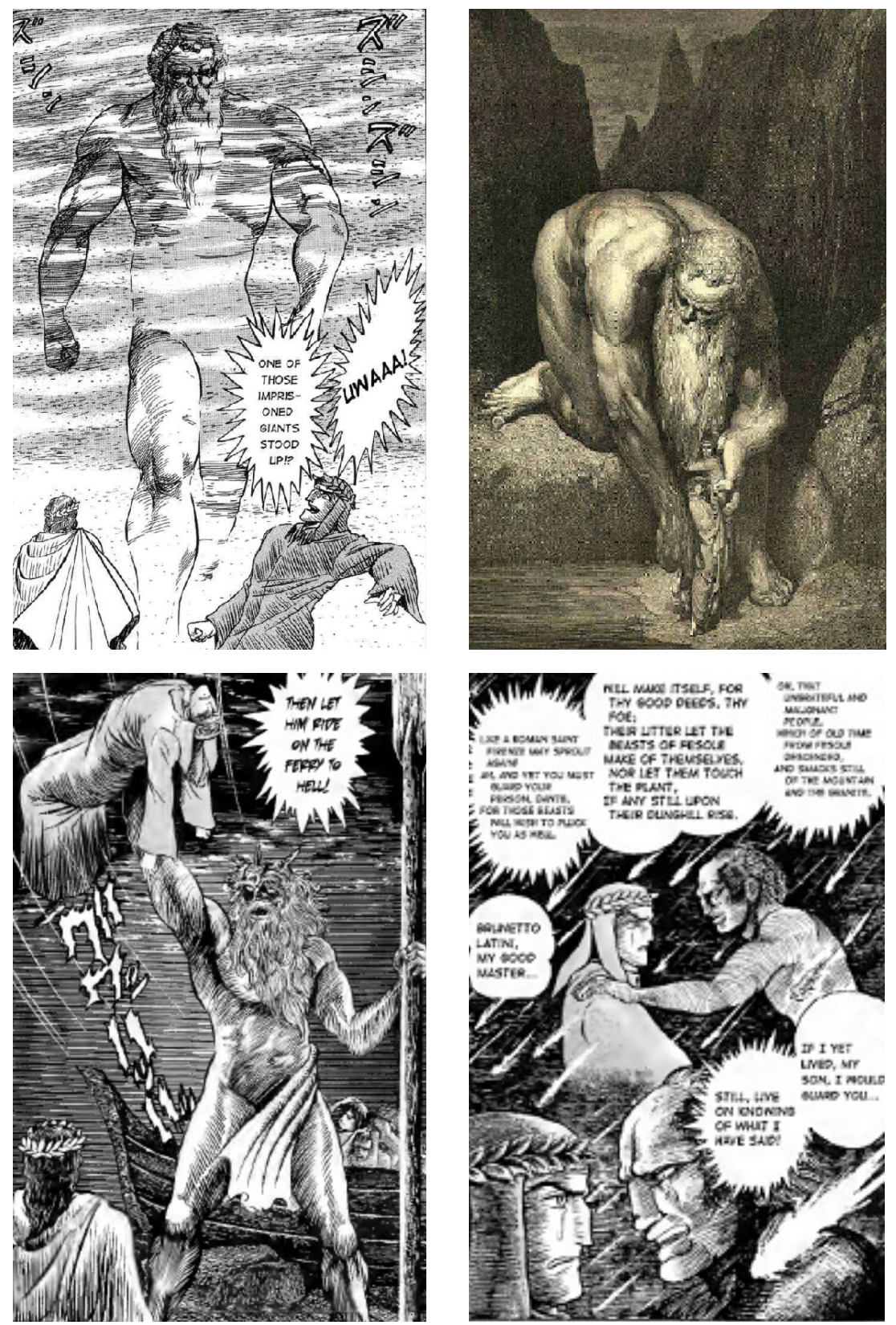

Figura 20. A sinistra la raffigurazione del gigante Anteo in Dante Shinkyoku (1994), a destra la corrispettiva illustrazione di Doré (I86I).

Figura 2I. Caronte solleva Dante, prima di scaraventarlo nella sua barca (Dante Shinkyoku, 1994). Figura 22. Dante incontra il suo maestro, Brunetto Latini (Dante Shinkyoku, 1994). 

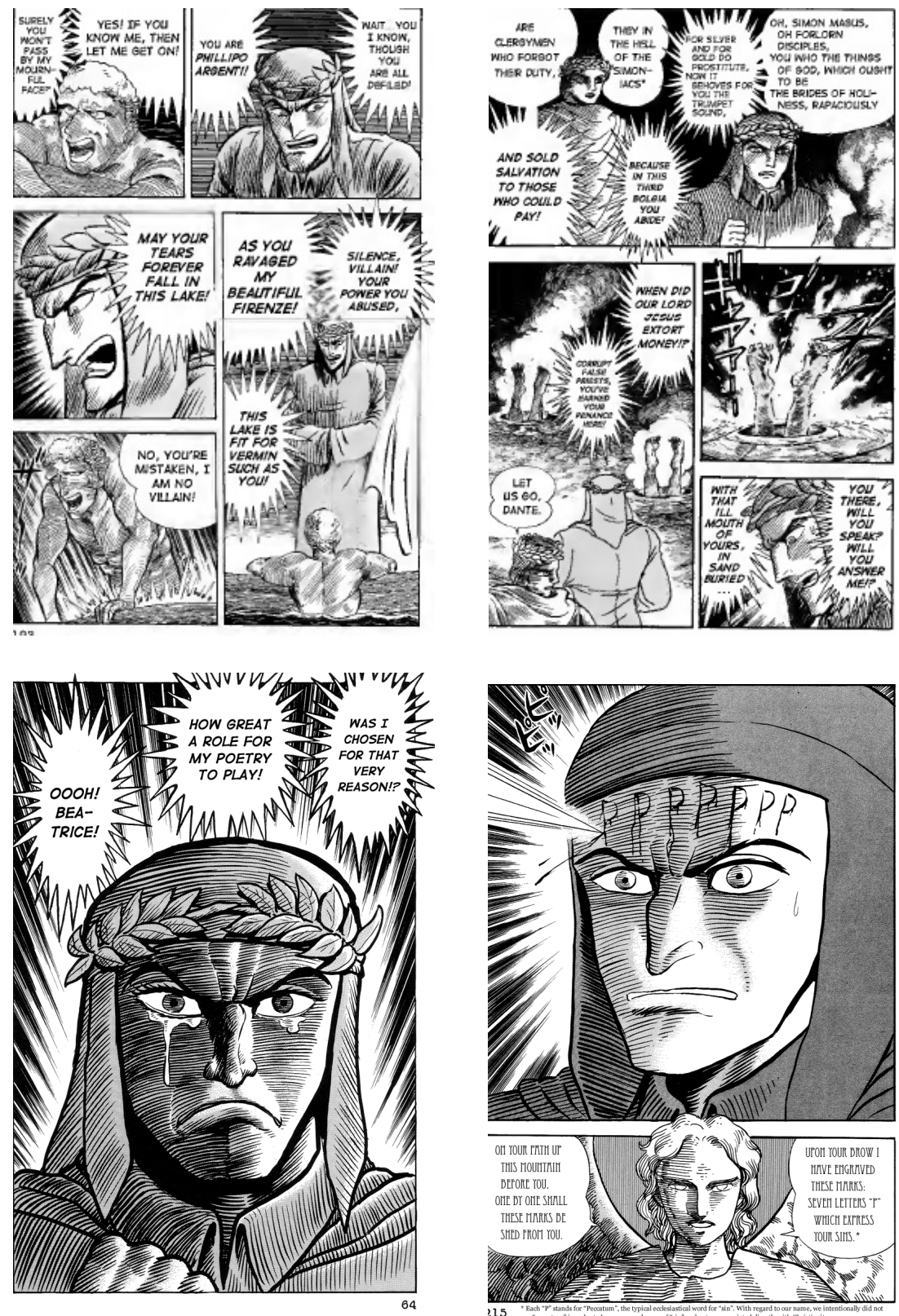

Figura 23a. Dante attacca Filippo Argenti (Dante Shinkyoku, 1994).

Figura 23b. Dante si rivolta contro i preti corrotti (Dante Shinkyoku, 1994).

Figura 24. Dante invoca Beatrice in lacrime (Dante Shinkyoku, 1994).

Figura 25. La fronte di Dante viene segnata con sette "P" (Dante Shinkyoku, 1994). 

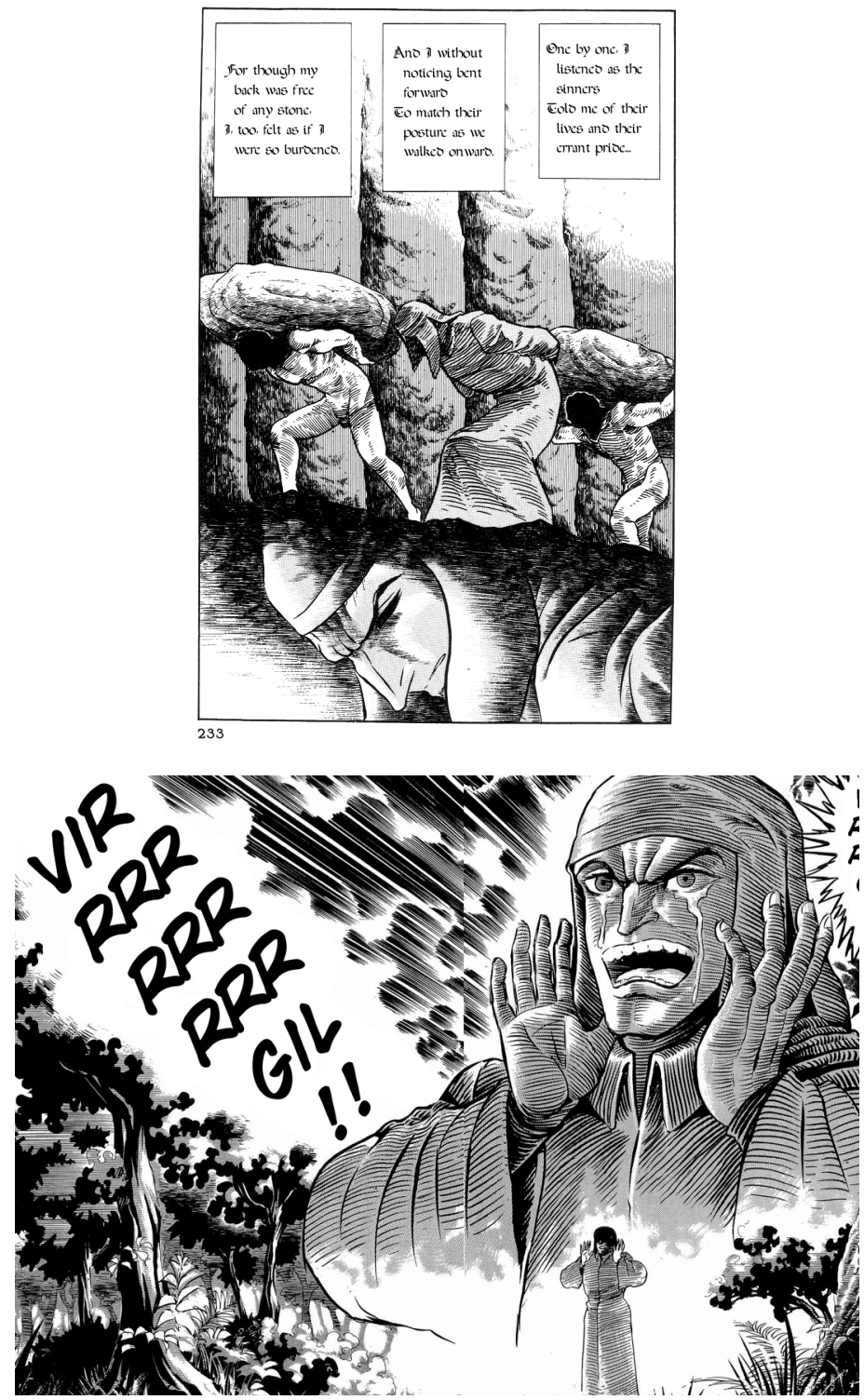

Figura 26. Dante curvo con i superbi (Dante Shinkyoku, 1994).

Figura 27. Dante urla il nome di Virgilio invano (Dante Shinkyoku, 1994). 

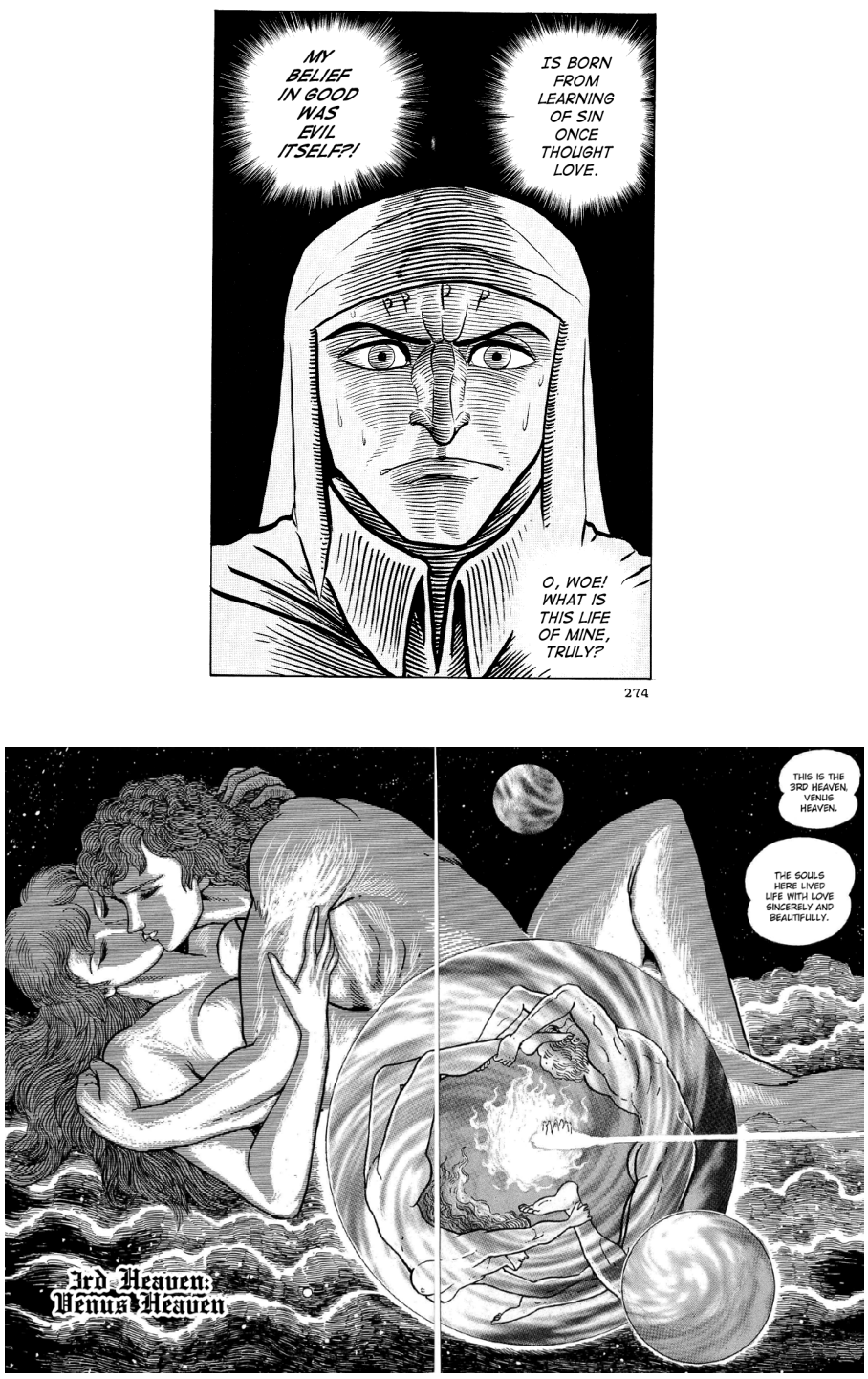

Figura 28. Dante è tormentato dai dubbi circa la bontà delle proprie scelte (Dante Shinkyoku, 1994).

Figura 29a. Paradiso. Il terzo cielo o Cielo di Venere (Dante Shinkyoku, 1994). 

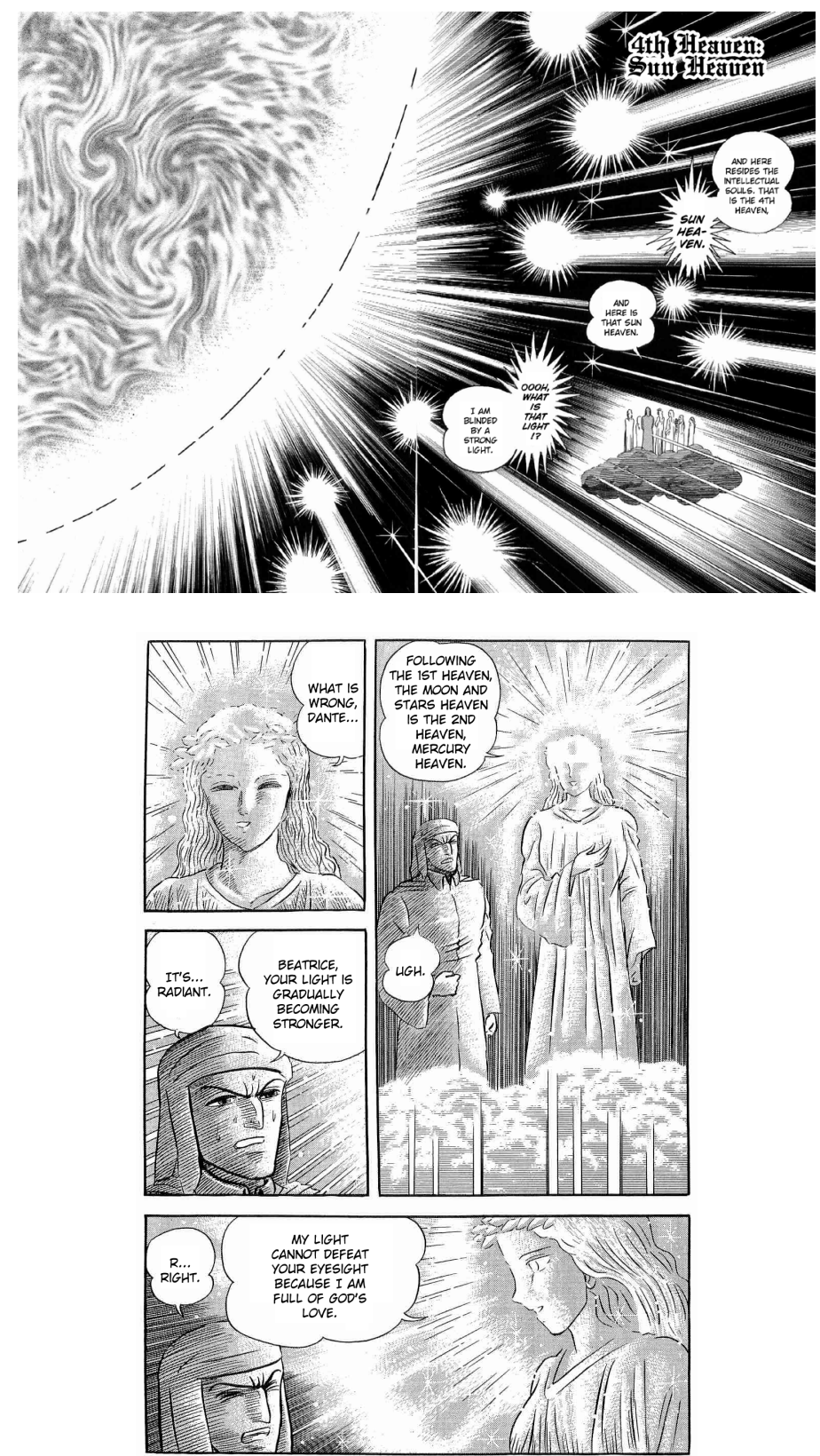

Figura 29b. Paradiso. Il quarto cielo o Cielo del Sole (Dante Shinkyoku, 1994).

Figura 30. Il tratto di Gō Nagai diventa sempre più abbagliante ed evanescente all'avvicinarsi alla luce di Dio (Dante Shinkyoku, 1994). 

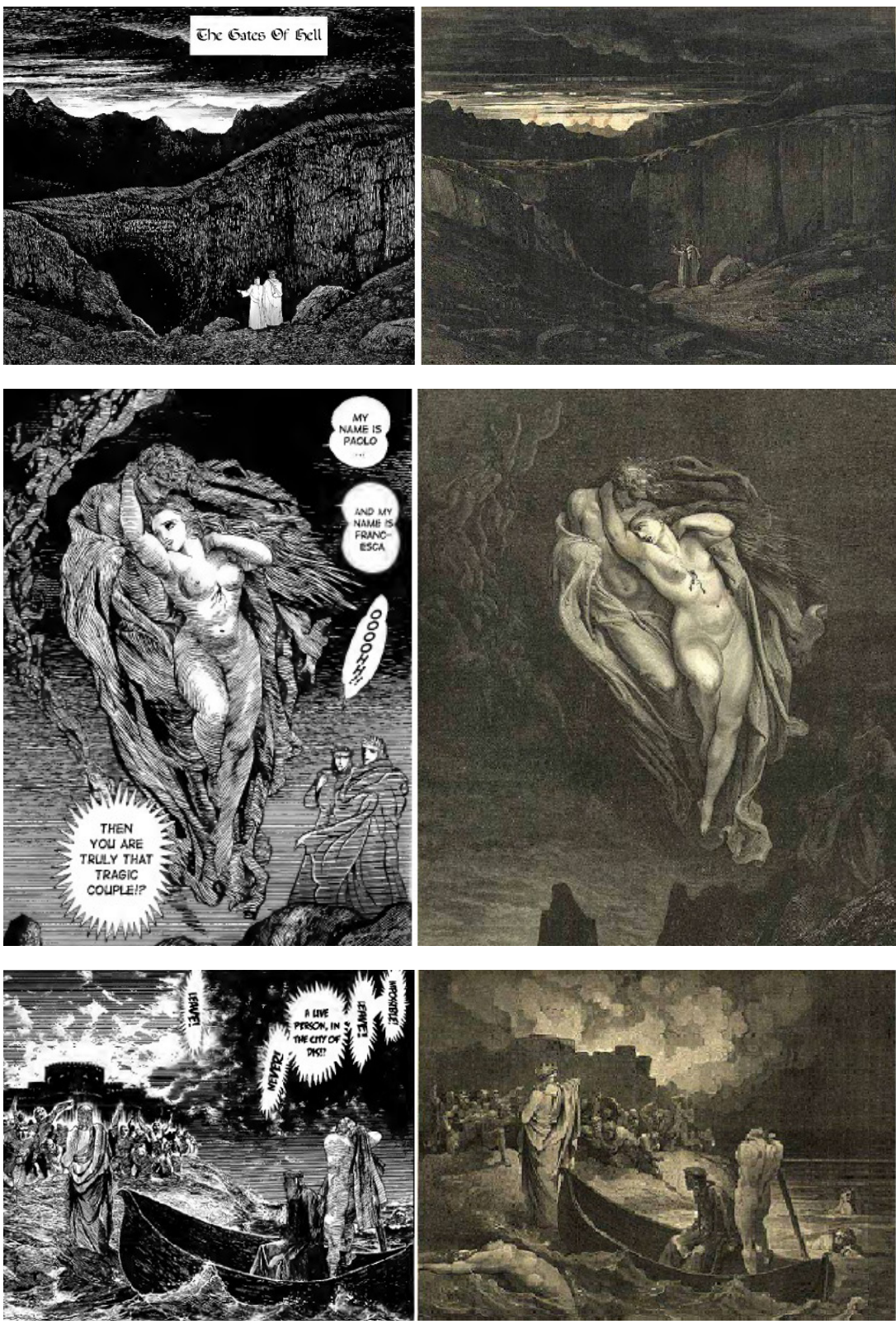

Figure $3 \mathrm{Ia}-3 \mathrm{Ib}-3 \mathrm{IC}-3 \mathrm{Id}-3 \mathrm{IC}-3 \mathrm{If}-3 \mathrm{Ig}-3 \mathrm{Ih}$. Alcuni esempi dell'incorporazione delle illustrazioni di Doré nel manga Dante Shinkyoku (1994). Per ciascuna immagine, a sinistra si trova la riproduzione di Gō Nagai, a destra l'incisione originale di Doré: Dante e Virgilio si avvicinano all'ingresso dell'inferno (3ra); Paolo e Francesca (3rb); Virgilio affronta i demoni fuori della città di Dite (3IC). 

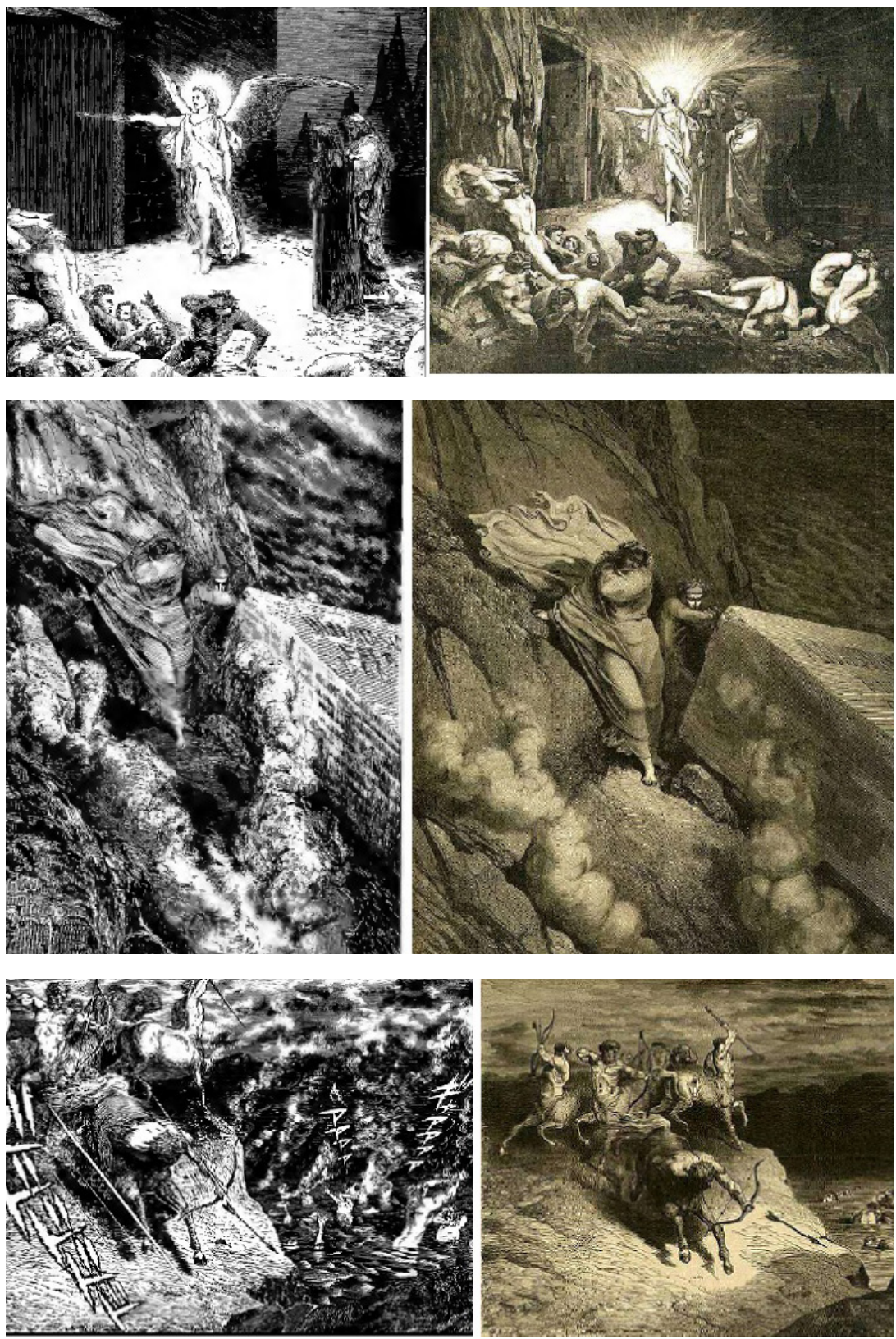

Il Messaggero celeste disperde i diavoli (3Id); Dante e Virgilio davanti alla tomba di Anastasio II (3Ie); i Centauri pattugliano i violenti contro gli altri (3If); Arpie nel bosco dei suicidi (3Ig); i demoni devono affrontare Dante e Virgilio (3rh). 

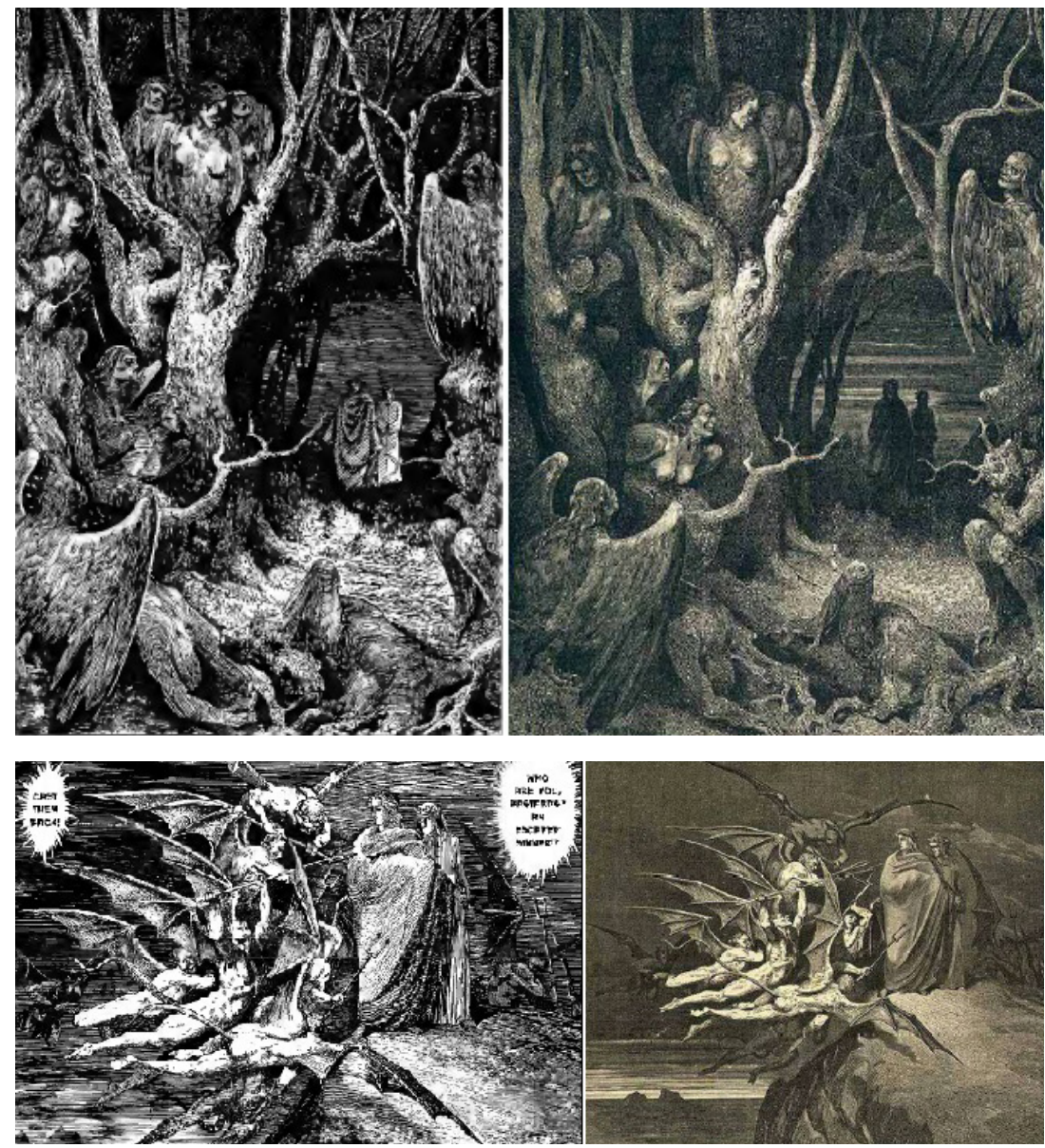

Arpie nel bosco dei suicidi (3Ig); i demoni devono affrontare Dante e Virgilio (3Ih). 

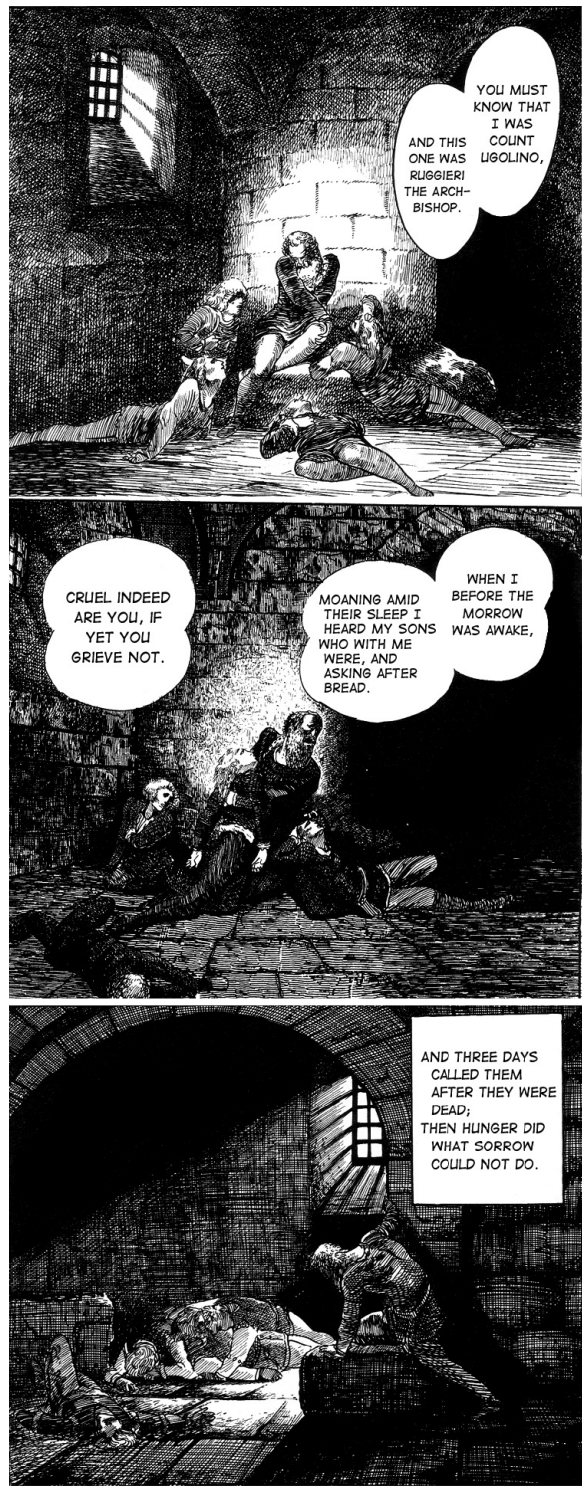

Figura 32. Dall'alto in basso le tre illustrazioni, tratte da Doré e riportate da Gō Nagai, per narrare la vicenda del Conte Ugolino (Dante Shinkyoku, 1994). 


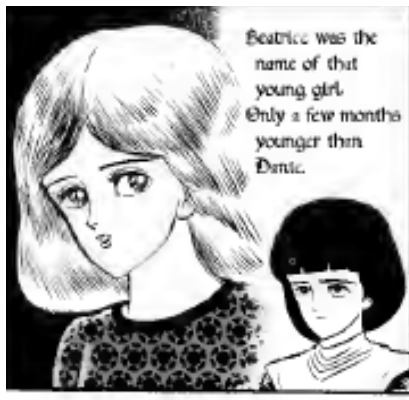

Anb from that

fils heart fast with Btart Dante knew heriecif movio strange articment A wotling votim of love at first Bight. beatungy

In awe Dente atoob before the matben.
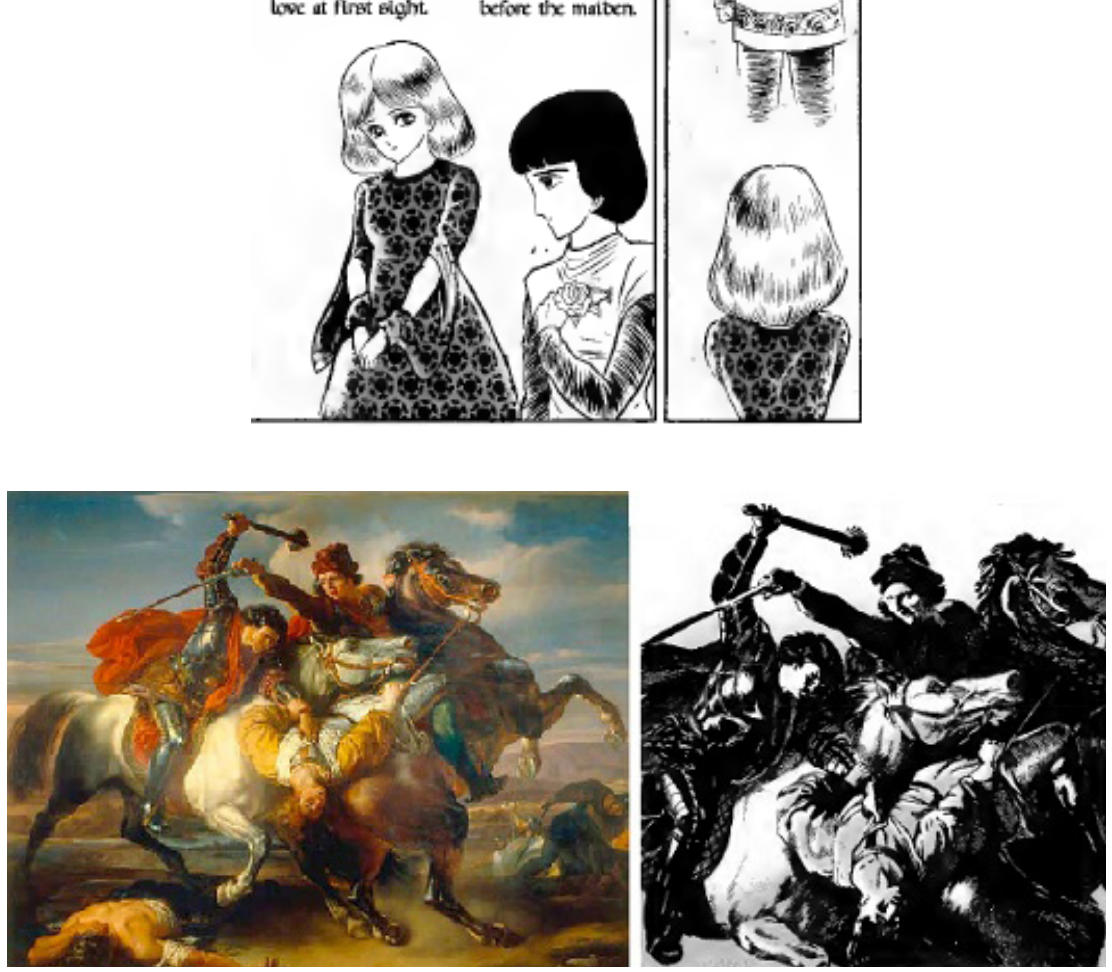

Figura 33. Excursus storico sul rapporto tra Dante e Beatrice, risalente all'infanzia (Dante Shinkyoku, I994).

Figura 34. A sinistra il dipinto Farinata degli Uberti alla battaglia di Montaperti (1842) di Giuseppe Sabatelli, a destra la riproduzione di Gō Nagai in Dante Shinkyoku (1994). 

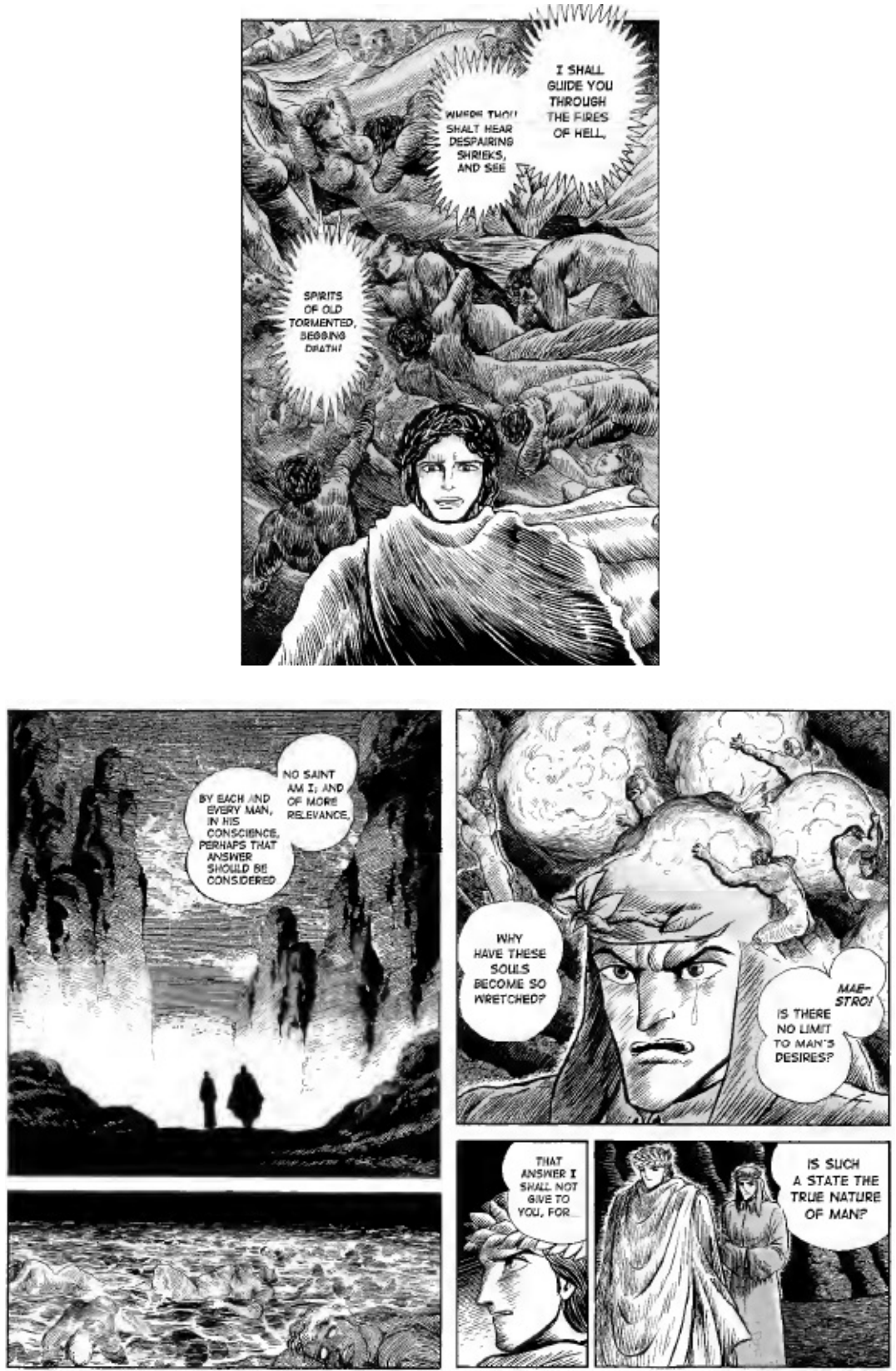

Figura 35. Le immagini evocate da Virgilio compaiono alle sue spalle nello spazio-schermo del fumetto (Dante Shinkyoku, 1994).

Figura 36. Rapidi cambi di inquadratura nella stessa pagina (Dante Shinkyoku, 1994). 